\title{
EMERGENCE AND EVOLUTION OF COOPERATION FOR SURVIVAL: A CONTINUOUS TIME MODEL
}

\author{
Delia Coculescu!, Oana Lupaşcu-Stamate $\mathrm{I}^{2}$ and Gabriele Visentin ${ }^{3}$
}

\begin{abstract}
For a large homogeneous population, where individuals rely on the availability of a resource for survival, we introduce a continuous time model for the availability of the resource in time and for each individual. In this framework, cooperation is defined as a mutual insurance mechanism, aimed at covering shortages for group members. We explore essential questions regarding the importance of cooperation: what are the characteristics of populations where cooperation is valuable, versus individualist populations, where cooperation destroys value; how large should be the groups of cooperating entities? In order to answer the latter question, we first characterise the optimal cooperation, which maximises the expected lifetime of entities in the population. Secondly, we explore the same question using a non cooperative stochastic game. This allows to understand to what extent strategic cooperation leads to inefficiencies as compared with optimal cooperation. We complete the theoretical results by suggestive numerical approaches.
\end{abstract}

KEYWORDS: risk sharing, mutual insurance, stochastic games in continuous time, inefficiencies of Nash equilibria, network formation.

\section{INTRODUCTION}

For a large homogeneous population where a non perishable good (that we call resource) is needed for survival, we propose a continuous time model for the availability of the resource in time and for each individual. In this framework, cooperation is defined as transfers of the resource between members in a given group, from those having a surplus towards those having a shortage of the resource. In other words, the notion of cooperation is to be understood in this paper as a mechanism of mutual insurance, where negative shocks (shortages of the resource) of some group members can be absorbed by positive shocks (surpluses) of other group members. Without any cooperation, an individual suffering from shortage of resource at a point in time is considered extinct from that time on. As cooperation creates dependencies between members, the evolution of the resource availability needs to be considered jointly, at the level of a group. By relying on the theory of Markov processes, we effectively measure the effects of cooperation on the expected lifetimes of group members. This allows to characterise the level of optimal cooperation, that leads to a maximal expected lifetime for group members. Achieving such an optimal level of cooperation would a priori require the presence of a regulator or central planner that forces groups of a certain size to form. The question

\footnotetext{
${ }^{1}$ University of Zurich, Department of Banking and Finance, Plattenstrasse 148032 Zürich, Switzerland. Email: delia.coculescu@bf.uzh.ch

${ }^{2}$ Institute of Mathematical Statistics and Applied Mathematics of the Romanian Academy, Calea 13 Septembrie 13

Bucharest, Romania. Email: oana.lupascu_stamate@ismma.ro

${ }^{3}$ University of Zurich, Institute for Mathematics, Winterthurerstrasse 1908057 Zürich 8032, Switzerland. Email: gabriele.visentin@math.uzh.ch
} 
that arises naturally is under what circumstances the population may reach by itself the optimal level of cooperation without such an intervention of a central planner, and to what extent inefficiencies appear.

To tackle this important question, we derive endogenously the size of cooperating groups, using a continuous time, non-cooperative stochastic game, where groups may decide to enlarge and include new individuals, with whom to cooperate from that point in time on, while exterior individuals decide on whether to join or not such groups. Once formed, a group follows the rules of cooperation without defection. Here, the underlying assumption is that members are linked by an agreement, be it formal or informal, that can be enforced $\left.\right|^{4}$ This assumption allows to focus our analysis solely on the process by which groups expand to include new members and to characterise the conditions where increasing the number of members is profitable. In our stochastic game, the actions of all players are based upon the observation of the state process summarising the need of resource at the level of an existing group. Importantly, we also account for the problem of adverse selection. For this, we introduce information asymmetry, and assume that the information about the state process of isolated individuals trying to join a group is only private to the individuals. Thus, a group that decides to expand, takes the risk to include a new member that has a deficiency of resource. Isolated individuals (exterior to a group) and the cooperating groups are assumed to be self-interested and aiming at maximising their respective expected lifetime. Assuming that the game evolves as Nash equilibria are played, we define the stable coalitions, as those groups that will never enlarge, because either they are not attractive enough for new members to join, or because they reject new members.

In this setting, we analyse the conditions under which cooperation emerges and how it evolves through time, leading either to the existence of stable coalitions (finite by nature) or infinite size coalitions. We then investigate whether strategic cooperation leads to inefficiencies as compared with optimal cooperation, that would be imposed by a central planner. We express inefficiencies both in terms of group size difference and of loss of expected lifetime, for wide ranges of the parameters, using a numerical approach.

The individuals in our model (that will be called entities) can be economic agents, households, production and supply units, financial institutions etc. By the intended generality of its formulation, the model we propose may apply in such diverse fields as biology, insurance, finance, management, economic and social exchange, whenever one can abstract from some specific phenomena such as social norms or kin selection in human populations.

1.1. Related literature and current contribution. In our approach, cooperation emerges because of an uneven distribution of resources through time and through the population. Cooperation therefore is nothing but a means of managing idiosyncratic risks related to the availability of the resource, based upon the idea of reciprocity. In this respect, our model is connected to some areas of research on risk sharing, or mutual insurance in networks. To the best of our knowledge, continuous time models of strategic network formation

${ }^{4}$ Informal arrangements may be enforced through a mix of quid pro quo, altruism (Cox [10]), or implicit social penalty costs (Besley and Coate [6]). Our model is also compatible with the interpretation that a given group has a joint liability to a third party, even though this is not explicitly modelled. Joint liability provides the necessary incentives for members to cooperate without defections. 
have not been considered so far in the literature. Our paper, in particular the game introduced in Section 4 is a continuous time model for strategic network formation, in a setting of a homogeneous population. Only complete networks are however considered, and such networks can be characterised by their size.

Some key modelling differences with the existing literature, that situate at the conceptual level will be discussed below, and we believe they complement the understanding of the value created by risk sharing. In a nutshell, we introduce the risk of extinction of populations with deficits of resource and cooperation is aimed at increasing expected lifetimes ${ }^{5}$ Also, we model for every individual the resource availability (as surplus, neutral or deficit), at the difference of models relying on wealth and consumption streams for each agent. This last aspect of our model may reveal useful in empirical applications, whenever complete data about revenues and consumption is difficult to obtain. Hence, our approach may be considered as a reduced-form model for mutual insurance.

Building on initial work of Bala and Goyal [4] modelling network formation as a non-cooperative game, several economists have studied informal transfers and their effectiveness in sharing risk. Kocherlakota [18], Kimball [17], Coate and Ravallion [8] or, more recently Ligon, Thomas, and Worrall [20] proposed economic models and show that risk sharing among non-altruists may occur through repeated interaction in repeated game models with self-interested agents, even without binding contracts. Generosity today is justified by expected future reciprocity. In large homogeneous populations, theoretical results suggest that the larger the population, the higher is the per capita utility from risk sharing, see for example Genicot and Ray [14]. This implies that a Pareto optimal solution to risk sharing for risk averse agents consists in forming as large a group as possible. For this reasons, risk sharing is in this literature called consumption smoothing, as its optimal outcome is that idiosyncratic risk is fully eliminated through mutual insurance.

Our work results in a more contrasted picture: we show that even when expected lifetimes are to be maximised by a central planner, individualistic populations do exist, where entities are better off without any form of cooperation, or sometimes small groups represent the optimal outcomes. Consequently, it may be optimal for subgroups of the large population to retain some idiosyncratic risk, by refusing an extended cooperation. Furthermore, we completely characterise the populations where finite optimal cooperation prevails (Proposition 3.6). We show that these populations are characterised by high levels of adverse selection risk, that exceed a given threshold. This threshold is shown to be a function of other parameters of the model, thus giving a clear picture of the tradeoffs taking place, between the implicit benefits and costs of cooperation.

These theoretical results are in line with numerous field evidence showing that smaller groups do better than larger groups with respect to risk sharing and full risk sharing is rare. For example, Deaton [11] (section 5.3) or research papers such as Ravallion and Dearden [26], Townsend [29], Udry [30], Grimard [15], Ravallion and Chaudhuri [25], Fafchamps and Lund [13], Murgai et al [23], Morduch [22] test for full consumption insurance at the village (community) level in developing countries. All of these papers

${ }^{5}$ In the case of households, extinction is to be interpreted as reaching a poverty trap and the expected lifetime refers to the average time spent outside the poverty trap. 
reject complete risk-sharing at the level of the community and find evidence of only partial cooperation. Some other existent theoretical results justify limited cooperation for risk sharing by frictions such as agents' impatience (Kocherlakota [18]), costs of developing links (Bramoullé and Kranton [7]) or heterogeneity of agents' preferences (Sadoulet [28], Attanasio et al. [3]). Barr and Genicot [5] explore the role played by intrinsic motivations, versus exterior constraints in reducing the sizes of cooperating groups.

In our model, the risks faced by the populations, such as the adverse selection when extending cooperation to new group members and the risk of extinction for a group, paired with the possibility of saving ressources as a protection agains negative shocks, are key in the outcome that finite groups are sometimes optimal. We present both theoretical results and numerical illustrations of the impact of these factors on the sizes of both Pareto optimal and strategically formed groups.

A founding assumption of our model is that agents save resources for later consumption. Saving is a mean to achieve consumption smoothing that is prevalent in all societies. We model the resource availability process exogenously; this may take the form of surpluses (savings) or deficits (shortages). Individuals possess a rate of surplus creation and depletion and an average time of survival in absence of a surplus. We shall derive explicit relations between these rates, that crucially impact the sizes of the Pareto optimal cooperating groups (Proposition 3.6) and of the strategic groups (Propositions 4.7 and 5.2). By introducing the notion of surplus in our model, the tradeoffs that agents face are similar to the ones that correspond to investment decisions under uncertainty, that is: keeping a safe asset (here consisting in keeping the surplus for own consumption in the future), versus undertaking a risky investment (here consisting in rescuing one distressed neighbour, that may be able to do the same later on). Some existing models consider endogenously the level of savings, as resulting from a decision making that is simultaneous with the consumption and risk sharing decisions (see Cole and Kocherlakota [9], Árahám and Laczó [1]).

The present work adds to the theory exploring risk sharing and mutual insurance, also by introducing some novel dimensions in the problem of optimal transfers.

First, we rely on the theory of Markov processes in continuous time in order to effectively characterise the behavior of cooperating groups, including long term evolution for infinite groups. Studying asymptotic properties is a key aspect in the frame of long term relations, with repeated interactions. Depending on the parameters, we show (Theorem 3.7) that in the limit of large groups, infinite cooperation leads either to population decline and eventual extinction, or at the opposite, resource accumulation leading to abundance and infinite lifetimes for everyone.

Second, for deriving results in a comprehensive form and avoid unnecessary complexity, we have made the choice to situate the model directly at the level of the resource availability, through the notions of surplus/deficit. The common approach in the literature so far has been to instead model revenues streams for the agents, or total wealths at a point in time. In our model, the total wealth or consumption of an agent is not specified, but instead we exogenously model the need of resource (as surplus, shortage of neutral). 
This conceptual difference with the literature is the one that makes possible to reach our objectives in the most simple and effective way.

Finally, another specific feature of our model as compared to the literature is that we introduce the risk of extinction. We consequently measure value in terms of expected lifetime rather than using utility functions or monetary values. By maximising expected lifetime, we perfectly capture agents' incentives without the need to incorporate total wealth, consumption and utility functions within the model. We believe that this modelling choice reinforces the applicability of our results, as in practice, utility functions, as well as total wealth or consumption streams of agents are difficult to estimate. The parameters of our model may be estimated from frequencies of observable events (such as acts of transfer, such that borrowing and lending, or occurrences of shortages, surpluses etc.) instead of the use of questionnaires involving subjective preferences. The scope of our model becomes thus more general and the results may be applied in other areas of risk management, not necessarily involving populations.

1.2. Outline of the paper. The paper is organised as follows. In Section 2 we propose a simple Markov chain model for describing the resource need of an entity without cooperation. We then proceed to introduce cooperation and describe how it affects the evolution of the individual needs of the members of the group and creates dependencies between the group members. In Section 3 , we compute explicitly the expected lifetime of a cooperating groups of size $N$ and derive the mathematical properties of the optimal expected lifetime. In terms of ranges of only three key variables, we can identify the populations that satisfy an optimal infinite cooperation, versus a finite one. We then turn in Section 4 to the modelling of the self-organisation process of entities in cooperating groups, using a continuous time strategic game and the notion of stable coalitions. We then analyse whether this process reaches by itself an optimal level of cooperation, without the intervention of a central planner. This topic is investigated both theoretically and using a numerical approach in Section 5. Section 6 offers some concluding remarks.

\section{THE MODEL}

We introduce a continuous time model to study the emergence of cooperation within a large, homogeneous population composed of entities that can be economic agents, households, production and supply units etc. The population relies on the availability of a certain resource for its survival. There is a state process for each entity that indicates the severity of its need of resource at a point in time, with three possible states: surplus, neutral and distress.

The entities evolve independently one from the other, unless they decide to cooperate and form a group. We shall call such a group a coalition, as the group forms specifically for the purpose of cooperating. Another reason for using this term, is that in the context of the strategic game in Section 4 coalitions will be considered to act together, as one unit, relative to the rest of the players When an entity is not part of a

${ }^{6}$ We do not use cooperative games in this paper, and coalitions here may not split, but only enlarge. 
coalition and it reaches the state of distress, nothing more happens and we can consider it as extinct. In other words, distress is an absorbing state for an entity in absence of cooperation. Inside a coalition on the other hand, each entity is linked to the others by relationships of borrowing and lending of the resource. These are aimed at improving the lifetime of the coalition's members, with entities in a surplus state ceding the surplus to the distressed entities. If all members of a coalition are distressed, entities cannot borrow the resource today or in the future and such a coalition is considered extinct.

To sum up, cooperation consists in relations of borrowing and lending the resource. It is not a priori clear that such a behaviour of cooperation is beneficial for all members, in that it increases their expected lifetime. Our aim is to explore this question.

2.1. The state process in absence of cooperation. We first consider a population where no lending and borrowing relations take place. The state process $\ell(k)=\left(\ell_{t}(k), t \geq 0\right)$ of a generic entity $k$ in this population is a stochastic process taking values in the set $\{-1,0,+1\}$, where

- state -1 indicates a shortage of resource or distress,

- state 0 indicates a normal level of the resource,

- state +1 indicates an excess of resource.

For simplicity, we will refer to "survival" state when an entity is in one of the states $\{0,+1\}$, hence not distressed.

We consider the population is made of independent and homogeneous entities, meaning, the processes $\ell(i)$ and $\ell(j)$ with $i \neq j \in \mathbb{N}^{*}$ are independent and have the same distribution. The state process of an entity is supposed to be a Markov chain with transition probabilities characterised by the following transition matrix:

$$
\mathbf{Q}^{(1)}=\left(\begin{array}{ccc}
0 & 0 & 0 \\
\alpha & -(\alpha+\gamma) & \gamma \\
0 & \delta & -\delta
\end{array}\right),
$$

where $\alpha>0, \gamma>0$ and $\delta>0$ are constants, the elements of the matrix containing the rates at which the process transitions from one state state to another. The exponent for $\mathbf{Q}^{(1)}$ is to emphasise that the matrix characterises the time evolution of a single entity and in absence of cooperation (that is, a coalition of size 1). Later on, we will use $\mathbf{Q}^{(N)}$ for the transition matrix corresponding to a coalition of size $N$.

We assume that any entity $k$ has the initial distribution

$$
\mathbb{P}\left[\ell_{0}(k)=-1\right]=p, \quad \mathbb{P}\left[\ell_{0}(k)=0\right]=(1-p) c, \quad \mathbb{P}\left[\ell_{0}(k)=+1\right]=(1-p)(1-c),
$$

with $c:=\frac{\delta}{\gamma+\delta}$ and $p \in(0,1)$.

This initial distribution is known under the name of quasi-stationary distribution. We refer to Méléard and Villemonais [21] for more details and an account of the relevance of this notion in the study of evolution of populations before extinction. From an economical perspective, an interpretation is that individual entities 
Key parameters:

- rate of the distress arrival (in absence of a resource surplus) $\quad \alpha>0$

- rate of surplus creation (in absence of a resource surplus) $\quad \gamma>0$

$\begin{array}{ll}\text { - rate of surplus depletion } & \delta>0\end{array}$

- probability of being distressed for a random entity (measure of adverse selection) $\quad p \in[0,1]$

- probability of being in the neutral state given survival $\quad c=\frac{\delta}{\gamma+\delta}$

- viability ratio

$\xi=\frac{\gamma}{\alpha}$

TABLE 1. Parameters characterising a random entity in absence of cooperation

have reached an equilibrium between the production and depletion of the resource: before they become extinct, they are able to keep a constant probability of having a surplus, without dependence on the time frame. This property is elementary: in absence of cooperation, an entity $k$ has a stationary conditional distribution given the entity is not distressed: for $t \geq 0$,

$$
\mathbb{P}\left[\ell_{t}(k)=i \mid \ell_{t}(k) \neq-1\right]=\overline{\boldsymbol{\mu}}(i), \forall i \in\{-1,0,+1\}
$$

with $\bar{\mu}:\{-1,0,+1\} \rightarrow[0,1]$ given by:

$$
\overline{\boldsymbol{\mu}}(-1)=0, \quad \overline{\boldsymbol{\mu}}(0)=c, \quad \overline{\boldsymbol{\mu}}(+1)=1-c .
$$

In Table 1 we summarise the parameters characterising a random entity. In our model, the level of resource that is available for one entity may be subject to both positive and negative shocks (in practice, these may be triggered either by unexpected levels of consumption or by varying revenues that are meant to support the consumption stream through time). We notice that negative shocks arrive at rate $\alpha$ when the entity has no surplus, or at the rate $\delta$ when the entity has a surplus. The case $\alpha>\delta$ is compatible with the existence of an implicit " interest", that is earned by the holder of a surplus, as negative shocks occur less often when possessing a surplus. The case $\alpha<\delta$ reflects implicit costs of holding a surplus of the resource. Also, from our analysis later on, it will appear that the viability ratio $\xi=\gamma / \alpha$ plays a crucial role in determining the level of cooperation.

The process characterised in (1) has the feature that it will be absorbed in finite time in the state -1 , hence an entity becomes distressed and extinct. While an entity is distressed, there are possibly other entities with a surplus. This raises the question of an allocation of the surplus of some entities toward other, distressed entities, with the scope of enabling them to recover from the distress state. In return, the entities ceding their surplus at one time may in the future benefit from the surplus of other entities in case of own distress. This is the basic idea of cooperation for survival. We next introduce a model for the effect of cooperation in a group of fixed size $N<\infty$. Afterwards, we will study the limiting behaviour when $N \rightarrow \infty$ and finally the size of a coalition will be derived endogenously, using a game-theoretic approach. 
2.2. Cooperation within finite groups. We generalise the previous model to describe the time evolution of the need of resource for members of coalitions, more exactly when $N \geq 1$ entities cooperate for survival. Hence, by taking $N=1$ we recover the model without cooperation. We shall call a coalition of size $N$ an N-coalition.

We define a multivariate stochastic process $\mathbf{L}^{(N)}$, hereafter named the resource availability process (or simply the state process) of the $\mathrm{N}$-coalition, that keeps track of the resource shortage or availability in a group of size $N$. More exactly,

$$
\mathbf{L}^{(N)}:=\left(L_{t}^{(N)}(1), \cdots, L_{t}^{(N)}(N)\right), t \geq 0,
$$

with each component $L_{t}^{(N)}(k)$ taking one of the values $\{-1,0,+1\}$ at time $t$ and representing the state of member $k$ of the coalition. For any member, we have an interpretation for the states as surplus, neutral and distress (same notation prevails as in the absence of cooperation, see Subsection 2.1).

The type of cooperation we aim at modelling can be described as follows. Let us take an element $\mathbf{x}=(x(i), i \in\{1, \cdots, N\}) \in\{-1,0,+1\}^{N}$ and consider that it describes the state of $N$ entities at time $t \geq 0$, that is, we have $\mathbf{L}_{t}^{(N)}=\mathbf{x}$. If member $i$ is being distressed (that is, $x(i)=-1$ ), then we assume it can borrow from any member in the group that has a surplus, that is every member $j$ with $x(j)=+1$. If there are several such possible lenders, we shall assume that one is chosen randomly among them. Once it is able to borrow, the state of entity $i$ turns from -1 to 0 and the state of its lender turns from +1 to 0 . If there is no entity with a surplus at that time, then there is no potential lender and member $i$ remains distressed. At a future time, members of the coalition may reach a surplus state and in this case lending and borrowing relations will resume, between one entity with a surplus and one distressed entity. We assume that except these borrowing lending relations there are no other interactions between the members of the coalition. This means that in between interactions, the state processes of entities evolve independently one from the other. Also, we will assume that entities are in an equilibrium as long as there are no distressed entities in the group (see Condition 2 below).

We notice that the mechanism we just described prevents an entity from being distressed as long as there are entities with surplus in the coalition, or, equivalently, an entity will persist in the distressed state -1 only as long as there are no entities with surplus in the coalition. The process $\mathbf{L}^{(N)}$ needs to satisfy:

$$
\text { If there exists } k \in\{1, \ldots, N\} \text { such that } L_{t}^{(N)}(k)=-1 \text {, then } \mathbf{L}_{t}^{(N)} \in\{-1,0\}^{N} \text {. }
$$

That means that the state process $\mathbf{L}^{(N)}$ is not visiting all the states in $\{-1,0,+1\}^{N}$.

Condition 1. The state space of the process. The state space of the process $\mathbf{L}^{(N)}$ is:

$$
I:=\left\{\mathbf{x} \in\{-1,0,+1\}^{N} \mid \text { if } \exists i \text { with } x(i)=-1 \text { then } x(j) \in\{-1,0\} \forall j \in\{1, \cdots, N\}\right\} .
$$

The next condition is the generalisation of the quasi-stationarity assumption for a single entity:

Condition 2. Distribution of the state process conditional on "zero distress". As long as there are no distressed members, that is $\mathbf{L}^{(N)} \in\{0,+1\}^{N}$, the distribution of the process $\mathbf{L}^{(N)}$ is stationary. 
The interpretation of Condition 2 is again in terms of an equilibrium: as long as no member of the coalition is distressed, all entities are able to keep a constant balance between creation and depletion of the surplus, as there is an expected frequency of being in a state of surplus that remains constant in time.

The reader may find in Appendix A.1 the mathematical definition of the state process $\mathbf{L}^{(N)}$ (Definition A.4) and the construction of a process satisfying Condition 1 and 2. This is given by a process with independent components that is reflected back into the set $I$ whenever it exits. The reflection occurs because existing surplus is transferred towards an entity with a deficit.

\section{EXPECTED LIFETIME WITH COOPERATION AND OPTIMAL SIZE OF COALITIONS}

We are interested in the expected lifetime of an entity, member of an $\mathrm{N}$-coalition. Because the state process $\mathbf{L}^{N}$ is a multivariate process with interdependent components, our approach is to rely on the distress level in a coalition instead, which is a simpler object.

3.1. The distress level in an $\mathbf{N}$-coalition. The distress level keeps track of the number of distressed entities at any point in time, without specifying the identities of these distressed entities:

Definition 3.1. (The distress level). The distress level of an $N$-coalition denoted as $Y^{(N)}$, is the stochastic process counting the number of distressed entities at any time $t$ within the $N$-coalition, that is:

$$
Y_{t}^{(N)}:=\left|\left\{k \in\{1, \cdots, N\} \mid L_{t}^{(N)}(k)=-1\right\}\right|,
$$

where we denote by $|A|$ the cardinality of a countable set $A$.

The distress level has the very convenient feature of being a Markov process and its distribution is characterised as follows:

Theorem 3.2. The distress level $Y^{(N)}$ of an $N$-coalition is a Markov chain with state space $\{0,1, \ldots, N\}$ and transition matrix $\mathbf{Q}^{(N)}=\left(q^{(N)}(i, j)\right)$, given by:

$$
q^{(N)}(i, j)= \begin{cases}\alpha N c^{N} & \text { if } i=0 \text { and } j=1, \\ \alpha(N-i) & \text { if } j=i+1 \text { and } i=1, \cdots N, \\ \gamma(N-i) & \text { if } j=i-1 \text { and } i=1, \cdots N, \\ 0 & \text { otherwise, with } i \neq j .\end{cases}
$$

The diagonal elements are as usual $q^{(N)}(i, i)=-\sum_{j \neq i} q^{(N)}(i, j)$.

Proof. See Appendix A.2.

We now comment on the implicit costs and benefits of cooperation for survival in our model; they result from comparing the properties of the distress level $Y^{(N)}$ with the properties of the process $\mid\{k \in$ $\left.\{1, \cdots, N\} \mid \ell_{t}(k)=-1\right\} \mid, t \geq 0$ that counts the number of distressed entities in a population of size $N$, 
but without cooperation (i.e., when the entities evolve independently one from the other). The effects of cooperation in a group of size $N$ are as follows:

- cooperation allows to spend more time in the state of no distress: if $Y^{(N)}=0$, the process is expected to remain in this state for a duration of $1 / \alpha N c^{N}$ while $N$ independent entities for a duration of $1 / \alpha c N$. This proves the the mutual insurance is very protective of distress.

- cooperation allows entities to recover from distress: there is a positive rate of recovery from distress of $\gamma(N-i)$ when $i$ entities are distressed. Alternatively, the recovery rate from distress is null for non cooperating entities.

- cooperation can potentially trigger faster cascades of distress: once there is non zero distress, e.g, $i \geq 1$ entities are distressed, the next distress in the coalition arrives at a rate $\alpha(N-i)$, while in a non cooperating group, it arrives at a lower rate of $\alpha c(N-i)$.

As we see, in addition of the convenient Markov property, the distress level $Y^{(N)}$ captures very well the effects of cooperation. Further, we are going to take the distress level as a state variable in our analysis of coalitions. First thing, we can now characterise the expected lifetime:

Definition 3.3. The expected lifetime of a member $i$ in an $N$-coalition containing $n \leq N$ distressed members is denoted by $h(n, N)$ and is defined as:

$$
h(n, N):=\mathbb{E}\left[\int_{0}^{\infty} \mathbf{1}_{\left\{L_{t}^{(N)}(i) \in\{0,1\}\right\}} d t \mid Y_{0}^{(N)}=n\right]
$$

for any $i \in\{1, \cdots, N\}$.

Remark 3.4. All entities in the coalition have the same distribution conditionally on the the information about the process $Y^{(N)}$, that is, the conditional distribution of $L_{0}^{(N)}(i)$ and $L_{0}^{(N)}(j)$ are identical. For this reason, the expected lifetimes of all entities in a coalition are equal.

Theorem 3.5. The expected lifetime of entity $i$ given that $Y_{0}=n$ is as follows:

(a) if $\alpha \neq \gamma$, then

$$
h(n, N)=h^{*}(n / N)+\frac{1}{\alpha N c^{N}}\left(1-\frac{c^{N}}{1-\xi}\right) \frac{\xi^{n}-\xi^{N}}{1-\xi},
$$

where $h^{*}(z):=\frac{1-z}{\alpha-\gamma}$ and $\xi:=\frac{\gamma}{\alpha}$;

(b) if $\alpha=\gamma$, then

$$
h(n, N)=\frac{1-n / N}{\alpha}\left(\frac{1}{c^{N}}+\frac{N+n-1}{2}\right)
$$

Proof. See Appendix A.3.

3.2. Optimal size of coalitions. We assume that a central planner chooses a group size $N^{*}$ (possibly infinite) that maximises the expected lifetimes of member entities. We also suppose that the central planner does not observe the state of an entity and choses randomly the entities from the entire population to form a 
coalition. When the size of a coalition is $N$, we assume that the distress level will satisfy $Y_{0}^{(N)} \sim \operatorname{Bin}(N, p)$, that is a binomial random variable with parameters $N$ and $p$, that counts the number of distressed entities in an $\mathrm{N}$-coalition at time $t=0$. We assume that $p<1$, since otherwise any $\mathrm{N}$-coalition is extinct at time $t=0$ and has a null expected lifetime. Under this assumption, we compute the optimal expected lifetime as:

$$
H^{*}:=\sup _{N \geq 1} \mathbb{E}\left[h\left(Y_{0}^{(N)}, N\right)\right]
$$

and we obtain:

Proposition 3.6. The following hold:

(a) If either $\frac{\gamma}{\alpha} \geq c$ with $p \in[0,1)$, or $\frac{\gamma}{\alpha} \in[0, c)$ with $p<\frac{1-c}{1-\frac{\gamma}{\alpha}}$, then the optimal expected lifetimes satisfy:

$$
H^{*}=\lim _{N \rightarrow \infty} \mathbb{E}\left[h\left(Y_{0}^{(N)}, N\right)\right]=\infty
$$

that is, the optimal coalition size is $N^{*}=\infty$.

(b) If $\frac{\gamma}{\alpha} \in[0, c]$ with $p \geq \frac{1-c}{1-\frac{\gamma}{\alpha}}$, then both the optimal expected lifetime $H^{*}$ and the optimal coalition size $N^{*}$ are finite. Further, it holds that

$$
H^{*} \geq \lim _{N \rightarrow \infty} \mathbb{E}\left[h\left(Y_{0}^{(N)}, N\right)\right]=h^{*}(p)=\frac{1-p}{\alpha-\gamma}<\infty .
$$

Proof. See Appendix A.4.

In Proposition 3.6, we characterise the parameters of populations where it is optimal to cooperate in infinite groups $\left(N^{*}=\infty\right)$, versus finite groups $\left(N^{*}<\infty\right)$. When $\frac{\alpha}{\gamma} \geq c$ infinite groups are always optimal, independently of the value of the parameter $p$. This means that as long that there is a non null fraction of surviving individuals to start with (given by $1-p$ ), even very small, it is possible for the distressed entities to recover through cooperation and achieve an infinite expected lifetime. But when $\frac{\alpha}{\gamma}<c$, the situation is different. The expected lifetime may be finite, if the fraction of distressed members is high initially at time $t=0$, and exceeds a certain threshold, given by:

$$
\pi:=\frac{1-c}{1-\xi} .
$$

We will refer to $\pi$ as the optimal probability threshold, as it results from determining the level optimal cooperation (as opposed to strategical cooperation, in the next section). We observe it increases with the viability ratio $(\xi=\gamma / \alpha)$ and with the probability to have a surplus, given survival $(1-c)$. This means that adverse selection becomes more tolerated when the viability is high and/or the probability of having a surplus given survival is high. Some illustrations are provided in Figures 1 and 2, where we can observe (for the case $\frac{\gamma}{\alpha} \leq c$ ), both convergence and divergence to infinity of the expected lifetime of N-coalitions, as $N$ increases, depending on whether $p<\pi$ or $p>\pi$.

A much finer understanding of the previous result is obtained when computing explicitly the time evolution of the proportion of distressed entities in infinite coalitions. This is analysed below. 

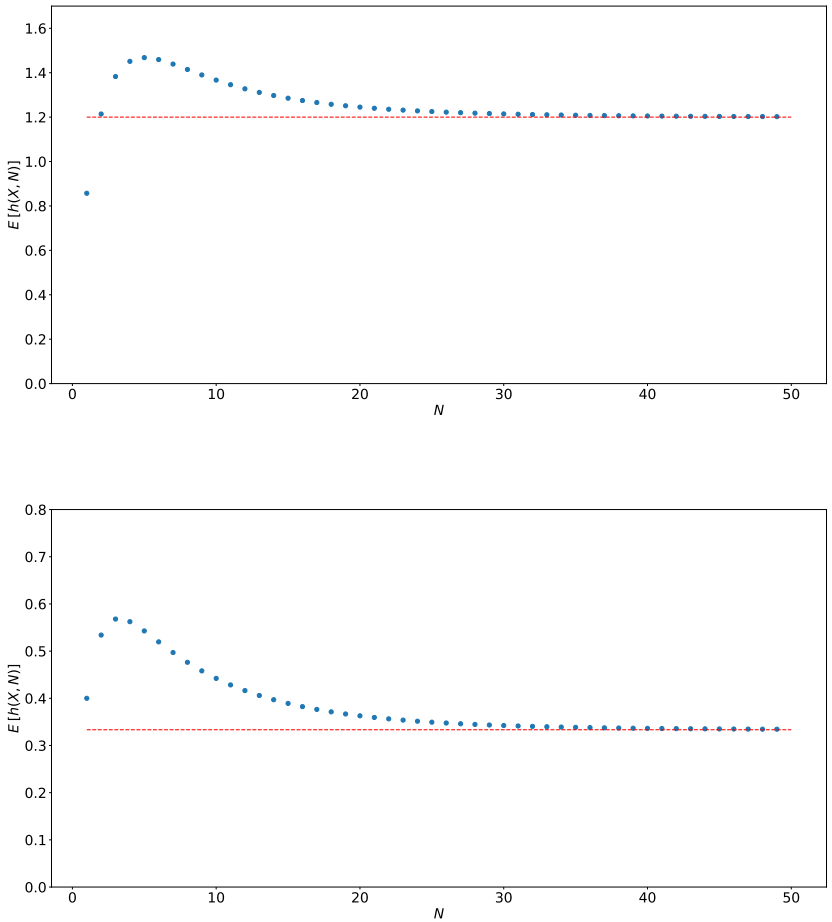

FIGURE 1. Convergence of the expected lifetime when the size of the coalition $N$ increases for $\frac{\gamma}{\alpha} \in[0, c]$ and $p \geq \frac{1-c}{1-\frac{\gamma}{\alpha}}$. The horizontal red line is the level $h^{*}(p)=\frac{1-p}{\alpha-\gamma}$. Above: $\alpha=0.5, \frac{\gamma}{\alpha}=0.4$, $c=0.5, p=0.4$. Below: $\alpha=0.5, \frac{\gamma}{\alpha}=0.4, c=0.7$ and $p=0.7$. The maximum expected lifetime $H^{*}$ is obtained with corresponding group sizes $N^{*}=5$ (above) and $N^{*}=3$ (below).

Theorem 3.7. The fraction of distressed entities in an $N$-coalition at time t:

$$
Z_{t}^{(N)}:=\frac{Y_{t}^{(N)}}{N}
$$

has the property:

$$
\lim _{N \rightarrow \infty} Z_{t}^{(N)}=Z_{t}
$$

where

$$
Z_{t}=1-\left(1-Z_{0}\right) e^{(\gamma-\alpha) t \wedge \tau}
$$

with $\tau=\inf \left\{t \mid Z_{t}=0\right\}$. Consequently, if $Z_{0}=0$ then $Z \equiv 0$. Otherwise if $Z_{0}>0$, we have:

(i) If $\frac{\gamma}{\alpha}>1$, then the process is absorbed at 0 after a deterministic time $\tau<\infty$.

(ii) If $\frac{\gamma}{\alpha}=1$, then $Z \equiv Z_{0}$.

(iii) If $\frac{\gamma}{\alpha}<1$, then $\lim _{t \rightarrow \infty} Z_{t}=1$. 

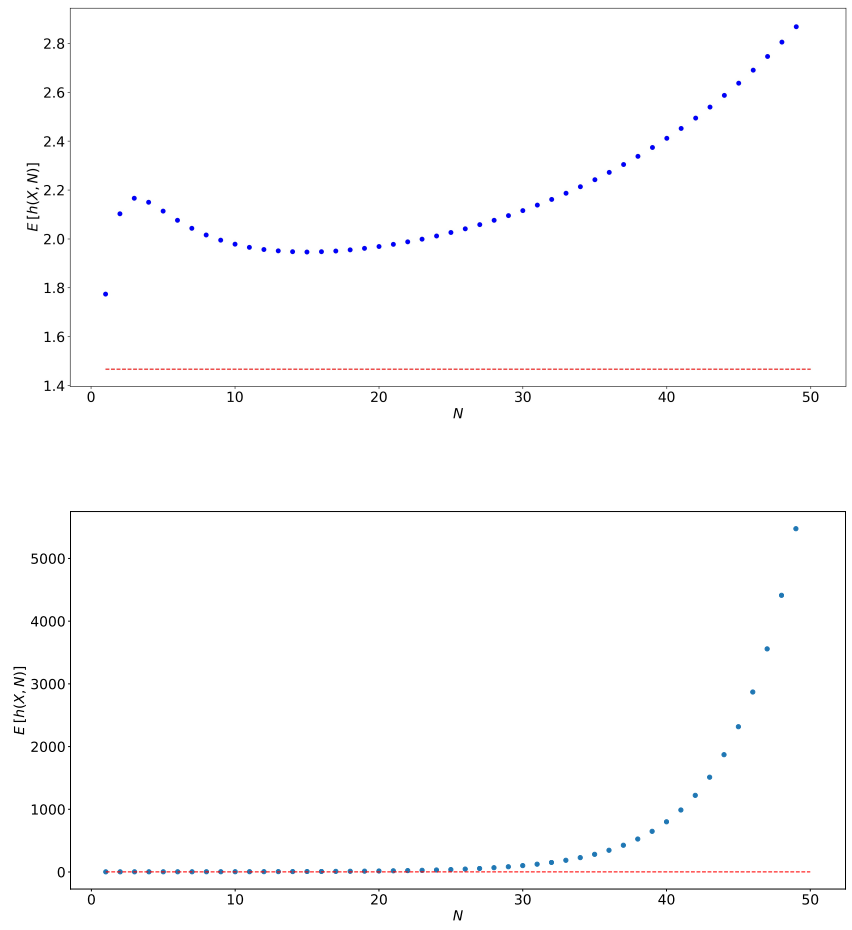

FIGURE 2. Divergence of the expected lifetime when the size of the coalition $N$ increases for $\frac{\gamma}{\alpha} \in$ $[0, c]$, with $p<\frac{1-c}{1-\frac{\gamma}{\alpha}}$. The horizontal red line is the level $h^{*}(p)=\frac{1-p}{\alpha-\gamma}$. Above: $\alpha=0.5, \frac{\gamma}{\alpha}=0.25$, $c=0.7, p=0.45$. Below: $\alpha=0.5, \frac{\gamma}{\alpha}=0.2, c=0.6$ and $p=0.3$. The optimal size of coalitions $N^{*}$ is infinite.

Proof. See Appendix A.5.

Considering still that $Y_{0}^{(N)} \sim \operatorname{Bin}(N, p)$, we obtain by the strong law of large numbers $Z_{0}=p$. Consequently, if $\frac{\gamma}{\alpha}>1$, by the finite time $\tau$ all distressed entities have recovered and there will be no more distressed individuals in the population ever after. The expected lifetime is indeed infinite in this case. The case $\frac{\gamma}{\alpha}=1$ corresponds to the situation where an infinite population will keep through time a constant proportion of distressed entities. Any distressed entity may recover and become distressed again later on, with a constant probability through time of being in one of these two situations. Finally, the case $\frac{\gamma}{\alpha}<1$ corresponds to the situation where the infinite coalitions become extinct in the limit, as $t \rightarrow \infty$. This complements the understanding of Proposition 3.6, as we see that the parameter $p$ does not change the final fate of such populations of becoming extinct asymptotically, nor it influences the rate at which such populations decline (which is given by $\alpha-\gamma>0$ ). Nevertheless, when $p$ is low enough, the entities can enjoy infinite expected lifetimes, as stated in Proposition 3.6(a). 
We may conclude that from the perspective of a central planner aiming at maximising expected lifetimes in populations, organising cooperation at the level of the whole population is an optimal policy whenever the conditions of Proposition 3.6 (a) are fulfilled, while cooperation in smaller groups of fixed size is optimal whenever the conditions of Proposition 3.6 (b) are fulfilled.

A crucial aspect at this point is to understand the self-organisation process of entities in cooperating groups and whether or not this process reaches by itself an optimal level of cooperation, without exterior intervention, as in the case of a central planner. This topic is investigated in the remaining of the paper.

\section{NASH EQUILIBRIA AND STABILITY OF COALITIONS}

We now turn to the study of the formation of coalitions within the frame of strategic game theory. This will allow us to understand to what extent the expected lifetimes will be improved when entities choose strategically to enter or not coalitions and coalitions also choose strategically whether to accept outsider entities and enlarge.

We assume naw that players choose strategies continuously in time, aiming to achieve the highest possible expected lifetime for themselves. We define the stable coalitions as those Nash equilibria that result in a coalition size remaining stable through time, independently of the state of distress of its members. Stable coalitions are those that have reached a certain size and their corresponding decision process will exclude new members ever after.

We aim at identifying the main drivers of stability of coalitions, in terms of the characteristics of the population, namely ranges of the parameters $\alpha / \gamma, c$ and $p$. The results of this section will be further enhanced by a numerical analysis in Section 5 .

4.1. Formalisation of the game. The game is represented by a sequence of stochastic non cooperative games $\Gamma=(\Gamma(N))_{N \geq N_{0}}$, with $N_{0}$ being a natural number. The stochastic games are played sequentially, one after the other: when $\Gamma(N)$ ends, $\Gamma(N+1)$ starts, unless the game $\Gamma(N)$ never ends, in which case the subsequent games will not be played. The game $\Gamma\left(N_{0}\right)$ starts at time $t=0$. The variable $N$ of $\Gamma(N)$ characterises the size that the coalition has reached, so that $N_{0}$ is the initial size of the coalition.

The game $\Gamma(N)$ corresponds to a game between a coalition of size $N$ and the "outside world", synthesised in player $\mathcal{P}_{\ominus}$, representing the proportion $p$ of distressed entities, and player $\mathcal{P}_{\oplus}$, representing the proportion $(1-p)$ of not distressed entities. Therefore, at any point in time there are three players to be modelled only. Even though the outside world contains an infinity of individuals, they only are of two types and it is sufficient to model only one representative of each type. For simplicity, we always consider $p \in(0,1)$, so that we do have the three players indeed.

All players choose actions continuously in time, while observing the state process of the N-coalition, $Y_{t}^{(N)}$, at any time $t$ for the duration of the game. Additionally, players $\mathcal{P}_{\ominus}$ and $\mathcal{P}_{\oplus}$ observe privately their own state, which is fixed through time (non stochastic). So, the state process for $\Gamma(N)$ is the Markov process $Y^{(N)}$. The N-coalition will expand to reach the size $N+1$ when the $\mathrm{N}$-coalition decides to include a new member; 
this new member will be chosen randomly among all those that played the action to accept to enter the $\mathrm{N}$-coalition at that point in time. Because the new member is randomly chosen, it is implicitly assumed that the $\mathrm{N}$-coalition does not observe the type of the new member whenever both players $\mathcal{P}_{\oplus}$ and $\mathcal{P}_{\ominus}$ are playing the strategy to join the coalition. This is the typical adverse selection problem (see Akerloff [2]). Indeed, with perfect information, the coalition would privilege the type $\mathbb{P}_{\oplus}$, so that the new member would not be randomly chosen. As soon as a new member is added, the game $\Gamma(N)$ stops and the game $\Gamma(N+1)$ continues from that point in time, with players being the coalition of size $N+1$ and the outside world, in a similar manner.

We now formalise our continuous time stochastic game (in a similar manner to Neyman [24]), as follows:

where:

$$
\Gamma(N)=\left(\left\{\mathrm{N} \text {-coalition, } \mathcal{P}_{\oplus}, \mathcal{P}_{\ominus}\right\}, \mathcal{I}^{(N)}, \mathcal{A}, \mathcal{H}^{(N)}, P^{(N)}, n_{0}^{(N)}\right), \text { for } N \geq N_{0}
$$

- $\left\{\mathrm{N}\right.$-coalition, $\left.\mathcal{P}_{\oplus}, \mathcal{P}_{\ominus}\right\}$ is the set of players.

- $\mathcal{I}^{(N)}=\{0,1, \cdots, N\} \times\{0\} \times\{1\}$ is the state space of the players. When the N-coalition is in state $n$ it means that it has $n$ distressed entities; the player $\mathcal{P}_{\oplus}$ has always 0 distressed entities, and finally, $\mathcal{P}_{\ominus}$ has always 1 distressed entity (being always in the distress state).

- $\mathcal{A}=\{a, b\}^{3}$ is the set of player's strategies (or actions). The set of actions remains the same, regardless of the size $N$ or state $n$ of the coalition.

- $\mathcal{H}^{(N)}:\{0,1, \cdots, N\} \times \mathcal{A} \times\left\{\mathrm{N}\right.$-coalition, $\left.\mathcal{P}_{\oplus}, \mathcal{P}_{\ominus}\right\} \rightarrow \mathbb{R}_{+}$is the payoff function, with values provided in the Table 2 below. Notice the use of a random variable $e(N)$ that stands for the type of the new member of the group, should the $\mathrm{N}$-coalition extend by action $(a, a, a)$. More details on this will follow below.

- $P^{(N)}=\left(\mathbf{Q}^{(N)}, \delta_{\{0\}}, \delta_{\{1\}}\right)$ are the transition probabilities of the three players. The transition matrix $\mathbf{Q}^{(N)}$ (that is given in Theorem 3.2 corresponds to the state process of the $\mathrm{N}$-coalition, the state of $\mathcal{P}_{\oplus}$ is constantly zero, and the state of $\mathcal{P}_{\ominus}$ is constantly one (by convention, so that the state 1 indicates one distressed entity).

- $n_{0}^{(N)} \in\{0, \cdots, N\}$ is the initial distribution for the N-coalition, at the time when the game $\Gamma(N)$ starts. This means that the state process $Y^{(N)}$ of the N-coalition takes an initial value $n_{0}^{(N)}$ at the time when the game $\Gamma(N)$ is initiated. The initial value will be endogenously determined within the game, except for the very first game $\Gamma\left(N_{0}\right)$, where it is exogenously specified. This will be further detailed below.

We introduce the following additional rules for the game $\Gamma(N)$, where $N \geq N_{0}$ :

(i) Strategy process. We suppose each player chooses actions among $\{a, b\}$, continuously in time, where $a$ stands for "accept" (agree to cooperate and extend the coalition) and $b$ for "block" (or not agree to extend the coalition, or not apply for becoming a member of the coalition). The decision (or strategy) process is a stochastic process $D_{t \in[T(N), T(N+1)]}$, adapted to the filtration generated by the state process $Y^{(N)}$, and taking values in $\mathcal{A}=\{a, b\}^{3}$, with $D_{t}$ being the action at time $t ; T(N)$ being the starting time (with $T_{N}=0$ if $N=N_{0}$ ) of the game and $T(N+1)$ being the stopping time of the game (defined below in (ii)). 


\begin{tabular}{c|l|l|l}
\hline \multicolumn{3}{|c}{ Payoff function $\mathcal{H}^{(N)}(n, s, i)$} \\
Player $i$ \\
$s \in \mathcal{A}$ & $i=\mathrm{N}$-coalition & $i=\mathcal{P}_{\oplus}$ & $i=\mathcal{P}_{\ominus}$ \\
\hline$(a, a, a)$ & $\mathbb{E}[h(n+e, N+1)]$ & $\mathbb{E}\left[h(n, N+1) 11_{\{e=0\}}+h(0,1) 11_{\{e=1\}}\right]$ & $\mathbb{E}\left[h(n+1, N+1) 11_{\{e=1\}}\right]$ \\
$(a, a, b)$ & $h(n, N+1)$ & $h(n, N+1)$ & 0 \\
$(a, b, a)$ & $h(n+1, N+1)$ & $h(0,1)$ & $h(n+1, N+1)$ \\
$(a, b, b)$ & $h(n, N)$ & $h(0,1)$ & 0 \\
$(b, a, a)$ & $h(n, N)$ & $h(0,1)$ & 0 \\
$(b, a, b)$ & $h(n, N)$ & $h(0,1)$ & 0 \\
$(b, b, a)$ & $h(n, N)$ & $h(0,1)$ & 0 \\
$(b, b, b)$ & $h(n, N)$ & $h(0,1)$ & 0 \\
\hline
\end{tabular}

TABLE 2. Values of the payoff function $\mathcal{H}^{(N)}(n, s, i)$, where $n \in\{0, \cdots, N\}$ is fixed. We consider $e \sim \operatorname{Bernoulli}(p)$, with $p \in(0,1)$.

(ii) Stopping rule. When the N-coalition is not extinct (that is $\left.Y_{t}^{(N)}<N\right)$, the game $\Gamma(N)$ is ended whenever $\mathrm{N}$-coalition plays $a$ and at least one type of players in the outside world plays $a$. Whenever the $\mathrm{N}$-coalition is extinct (that is $Y_{t}^{(N)}=N$ ), we consider the game $\Gamma(N)$ continues, unless player $\mathbb{P}_{\oplus}$ steps in to rescue (that is, $\mathrm{N}$-coalition plays $a$ and $\mathbb{P}_{\oplus}$ plays $a$ ). By this, we avoid studying enlargements of extinct coalitions that only lead to extinct coalitions. Therefore, the ending time of the game $\Gamma(N)$ is defined as:

$$
T(N+1):=\inf \left\{t \geq T(N) \mid \text { either: } Y_{t}^{(N)}<N, D_{t} \in E \text { or: } Y_{t}^{(N)}=N, D_{t} \in E^{\prime},\right\}
$$

with $E=\{(a, a, a),(a, b, a),(a, a, b)\}$ and $E^{\prime}=\{(a, a, a),(a, a, b)\}$. We use the convention inf $\emptyset=+\infty$. Remark that $T(N)$ is a stopping time in the filtration generated by the state process $Y^{(N)}$.

(iii) The payoff function. Table 2 summarises the payoff function at time $t \in[T(N), T(N+1))$ for each player, depending on:

- the state of coalition at time $t$, given by the state of process $Y_{t}^{(N)}=n \in\{0,1, \ldots, N\}$,

- the action taken by the players at time $t, D_{t} \in \mathcal{A}$.

As there are finitely many states, players and strategies, that table is exhaustive of the payoffs.

The payoffs make use of the function $h$, that is the expected lifetime in coalitions. Whenever the actions are not in $E$, players have a payoff that equals their own expected lifetime. When actions belong to the set $E$, the coalition may expand to include a new member and therefore the payoffs depend on the state of the new member $e(N) \in\{0,1\}$. This new member is assumed to be randomly chosen among the outside entities that play $a$. So, for actions that belong to the set $E$, the payoff for the coalition and for the new accepted member is $h(n+e(N), N+1)$. For instance, if action $(a, a, a)$ is played, the coalition chooses one new member at random from the general population, hence $e(N)$ is Bernoulli with parameter $p$ (simply denoted by $e$ in Table 2). As $e(N)$ is independent from the filtration of the process $Y^{(N)}$, but the decision $D$ must be adapted to it, we project the payoff on the filtration of the process $Y^{(N)}$. In this case, strategies 
are decided by players upon comparing expected values.

In the cases where action $(a, b, a)$ or $(a, a, b)$ are played, only one type within the outside population is willing to join the $\mathrm{N}$-coalition, so that $e(N)$ equals the state of this player (hence is not random).

(iv) Starting rule. At $t=T(N+1)$ the game $\Gamma(N+1)$ starts with initial value

$$
n_{0}^{(N+1)}:=Y_{T(N+1)}^{(N)}+e(N) .
$$

As the state process $Y^{(N)}$ is Markov, we will focus on Markov strategies only, that are only dependent on the current state. That means that we will impose the stronger requirement that the strategy process $\left(D_{t}\right)_{t \in[T(N), T(N+1))}$ satisfies the property: $\forall t \geq 0, D_{t}$ is a function of $Y_{t}^{(N)}$. Requiring the strategies to be functions of the current state of the process $Y^{(N)}$ is very useful, as it permits to analyse the game $\Gamma(N)$ through the collection of sub-games $\Gamma(n, N), n \in\{0, \cdots, N\}$, with each sub-game $\Gamma(n, N)$ being a non-stochastic game, corresponding to payoff $\mathcal{H}^{(N)}(n, s, i), s \in \mathcal{A}$ and $i \in\left\{\mathrm{N}\right.$-coalition, $\left.\mathbb{P}_{\oplus}, \mathbb{P}_{\ominus}\right\}$.

A fundamental concept for non zero sum games is the Nash equilibrium. A Nash equilibrium for $\Gamma(N)$ at time $t$, given that $Y_{t}^{(N)}=n$, is a Nash equilibrium for the sub-game $\Gamma(n, N)$. The notion of stability of coalitions is introduced below:

Definition 4.1. (i) An N-coalition is stable if, when Nash equilibria strategies are played in each point in time, the game $\Gamma(N)$ is never ending (that is if $N_{0}=N$ then $T(N+1)=\infty$ ).

(ii) A coalition that is not stable is said to be unstable.

Remark 4.2. Given the expression in (5), an N-coalition is stable if:

- for any $Y_{t}^{(N)}<N$, there are not Nash equilibria in $E=\{(a, a, a),(a, b, a),(a, a, b)\}$ and,

- for $Y_{t}^{(N)}=N$, there are not Nash equilibria in $E^{\prime}=\{(a, a, a),(a, a, b\}\}$.

4.2. An analysis of stability of coalitions. In order to analyse stability of coalitions, we will be assuming in this subsection that players choose their strategies only among the Nash equilibria, at each point in time and for any size $N$ and state $n$ of a coalition prevailing at that point in time. We will prove the existence of stable coalitions and characterise some generic conditions for stability versus instability.

First, we characterise stability of N-coalitions in terms of inequalities involving the payoff functions of the players.

Proposition 4.3. An N-coalition is stable if the following conditions are fulfilled:

(i) for all $n \in\{0, \ldots, N-1\}$,

$$
h(n, N)>h(n+1, N+1) ;
$$

(ii) for all $n \in\{0, \ldots, N\}$ one of the following holds:

$$
\begin{aligned}
h(0,1) & >h(n, N+1) \\
\text { or } \quad h(n, N) & >p h(n+1, N+1)+(1-p) h(n, N+1) .
\end{aligned}
$$


Proof. Using the values of the payoff function $\mathcal{H}^{(N)}$ written in Table 2, we need to prove that:

1. the condition (6) together with either (7) or $(8)$, for $n \in\{0, \ldots, N-1\}$ are equivelant to: there are not Nash equilibria in $E=\{(a, a, a),(a, b, a),(a, a, b)\}$ for the corresponding sub-game $\Gamma(n, N)$;

2. the condition either (7) or (8), for $n=N$ is equivalent to: there are not Nash equilibria in $E^{\prime}=$ $\{(a, a, a),(a, a, b)\}$ for the corresponding sub-game $\Gamma(N, N)$.

We prove the point 1 . First, we observe that $(a, a, b)$ is never a Nash equilibrium for $n<N$. Intuitively, distressed players have always a strict preference to enter a coalition as this action provides them a chance to be rescued. One can notice by inspection of the payoff function $\mathcal{H}^{(N)}$ if a non extinct $\mathrm{N}$-coalition plays $a$, the player $\mathbb{P}_{\ominus}$ will always play $a$, as the corresponding payoffs strictly dominates the payoff 0 , that would be obtained by playing $b$.

Condition (i) is the equivalent of: the action $(b, b, a)$ delivers a strictly higher payoff for the $\mathrm{N}$-coalition than $(a, b, a)$ and this, for all $n \in\{0,1 \ldots, N-1\}$, so that $(a, b, a)$ is not a Nash equilibrium in the corresponding sub-games ${ }^{7}$

Condition (ii) for $n \in\{0, \cdots, N-1\}$ is the equivalent of: either the action $(b, a, a)$ delivers a strictly higher payoff for the $\mathrm{N}$-coalition than action $(a, a, a)$, or the action $(a, b, a)$ delivers a higher payoff for $\mathbb{P}_{\oplus}$ than action $(a, a, a)$. In either case, $(a, a, a)$ is a not a Nash equilibrium for the sub-games $\Gamma(n, N), n \in\{0, \cdots, N-1\}$.

Now, we prove the point 2. Focussing specifically on condition (ii) and the case $n=N$ we find that (8) is not fulfilled. So that condition (ii) requires that (7) holds true. But this implies that player $\mathbb{P}_{\oplus}$ has a strict advantage in playing $b$. Consequently neither strategies in $E^{\prime}$ are Nash equilibria.

It is useful to take the point of view of the healthy outsiders, that is, player $\mathcal{P}_{\oplus}$. It will play $b$ if $h(n, N+1)<h(0,1)=\frac{1}{\alpha c}$. Given that the mapping $n \mapsto h(n, N)$ is decreasing (see Lemma A.7), there is a critical level of distress, denoted $n^{\text {out }}(N)$ such that for $n \in\left\{0, \ldots, n^{\text {out }}(N)\right\}, \mathcal{P}_{\oplus}$ plays $a$ and for $n>n^{\text {out }}(N)$ $\mathcal{P}_{\oplus}$ never plays $a$ (too much distress). We call this level the critical level of distress.

Definition 4.4 (Critical level of distress for an N-coalition). For $N$ finite we define

$$
n^{\text {out }}(N):=\max \left\{n \in\{0, \ldots, N\} \mid h(n, N+1) \geq \frac{1}{\alpha c}\right\} .
$$

As the mapping $n \mapsto h(n, N+1)$ is decreasing, $n^{\text {out }}(N)$ is well-defined and $n^{\text {out }}(N) \in\{1, \ldots, N\}$. Indeed, $h(0, N+1)>h(0,1)=\frac{1}{\alpha c}$, so that $n^{\text {out }}(N)>0$ and $h(N+1, N+1)=0<\frac{1}{\alpha c}$ so that $n^{\text {out }}(N) \leq N$.

Lemma 4.5. If $n^{\text {out }}(N)=N$, then an $N$-coalition is unstable. Non distressed outsiders always want to enter such a coalition, regardless of its state of distress.

\footnotetext{
${ }^{7}$ We do not impose the condition (i) for $n=N$ because we know that there it is not satisfied. We have indeed: $h(N, N)=h(N+1, N+1)=0$. This means that an extinct $N$-coalition will be indifferent between action $(a, b, a)$, that is, enlarging with a distressed entity, or action $(b, b, a)$, that is, block the entrance of a distressed entity. But both actions lead to an extinct coalition.
} 
Proof. Suppose that the N-coalition is extinct, that is $n=N$, then $h(N, N)=0$. Also, because $n^{\text {out }}(N)=N$ we have $h(N+1, N)>h(0,1)$. Therefore, condition (ii) in Proposition 4.3 is not satisfied and the Nash equilibrium is $\{a, a, a\}$. The coalition extends to incorporate a new member.

In the next proposition we give a characterisation of a stable $\mathrm{N}$-coalition using the critical level of distress $n^{\text {out }}(N)$, that will reduce the number of inequalities to check (indeed Proposition 4.3 contained some overlapping conditions).

Proposition 4.6. An N-coalition is stable if and only if the following hold:

(i) for all $n \in\left\{0, \ldots, n^{\text {out }}(N)\right\}$ we have:

$$
p>\frac{h(n, N+1)-h(n, N)}{h(n, N+1)-h(n+1, N+1)} .
$$

(ii) If $n^{\text {out }}(N) \leq N-2$, then for all $n \in\left\{n^{\text {out }}(N)+1, \ldots, N-1\right\}$ we have:

$$
h(n, N)>h(n+1, N+1) .
$$

Proof. The assertions are direct consequences of Proposition 4.3 and Definition 4.4. Lemma A.7justifies the order of the inequalities (9), the denominator of the fraction being strictly positive.

We now give a first result on the stability of coalitions. This result is general, as it does not involve the probability $p$ that is, the proportion of distressed entities in the "outside world" measuring the risk of adverse selection. It will be followed by an analysis involving the role or the probability $p$, in the next section.

\section{Proposition 4.7. The following assertions hold:}

(i) If $\frac{\gamma}{\alpha} \geq 1$, then any $N$-coalition is unstable: $n^{\text {out }}(N)=N$ for all $N$.

(ii) If $\frac{\gamma}{\alpha} \geq c$, then there are not arbitrarily large stable $N$-coalitions: there exists $N^{*}$ such that $n^{\text {out }}(N)=N$ for all $N \geq N^{*}$.

(iii) If $\frac{\gamma}{\alpha} \in(0, c)$, then $n^{\text {out }}(N)<N$ for all $N$.

Proof. We will use the representation (23) of the function $h$, that implies:

$$
h(N, N+1)=\frac{1}{\alpha c(N+1)}\left((\xi / c)^{N}+\sum_{j=0}^{N-1} c(\xi)^{j}\right) .
$$

(i) By Lemma 4.5, to show that the N-coalition is not stable, it suffices to show that $h(N, N+1) \geq \frac{1}{\alpha c}$, that is $n^{\text {out }}(N)=N$ (the healthy outside entities are always willing to join the $N$-coalition). Using (11) and $\frac{\gamma}{\alpha} \geq 1$, we obtain: $h(N, N+1) \geq \frac{1}{\alpha c}\left(\frac{1+N c^{N+1}}{(N+1) c^{N}}\right)>\frac{1}{\alpha c}$, because $\frac{1+N c^{N+1}}{(N+1) c^{N}}>1$, this being equivalent to $c<1$ (and this condition on $c$ being always satisfied, see Table 1).

(ii) If $\frac{\gamma}{\alpha} \geq c$, then $\lim _{N \rightarrow \infty} h(N, N+1)=+\infty$, so that there is $N^{*}$ so that $h(N, N+1)>\frac{1}{\alpha c}$ for all $N \geq N^{*}$. 
(iii) We have $n^{\text {out }}(N)<N$ if and only if $h(N, N+1)<\frac{1}{\alpha c}$. Or, this inequality can be checked easily, using $\frac{\gamma}{\alpha}<c$ in $11,: h(N, N+1)<\frac{1}{\alpha c} \cdot \frac{N+1}{N+1}=\frac{1}{\alpha c}$.

Proposition 4.7 implies that if stable coalitions exist, they must have the feature that the viability ratio $\xi=\gamma / \alpha$ is below 1 . When $\xi \geq 1$, infinite coalitions form as healthy outsiders are always ready to rescue even the extinct coalitions. This is consistent with the results on optimal cooperation, as we have seen that a viability ratio of at least 1 implies infinite lifetimes for coalitions with infinitely many members (Theorem 3.7). We may conclude that entities acting strategically achieve an infinite lifetime also without a central planner when $\xi \geq 1$. Recall that Proposition 3.6 indicates that optimal cooperation that is finite exists only in the case $\xi \leq c$. Proposition 4.7 suggests that inefficiencies of strategic cooperation may exist: indeed, stable coalitions may exist also for $\xi \geq c$ when $N$ low, because for small-sized coalitions there may exists a level of distress such that it becomes unattractive to be entered by healthy outsiders (that is, $\left.n^{\text {out }}(N)<N\right)$ ). This is only a necessary condition for stability, but not sufficient one. To obtain sufficient conditions, we need to examine the role of the level of adverse selection, $p$, and this is analysed next. Simply stated, the role of $p$ will be to partition the populations in stable/unstable for the case $\frac{\gamma}{\alpha}<1$, when $N$ is fixed.

\section{BOUNDARIES OF STABILITY AND NUMERICAL ILLUSTRATIONS}

5.1. Characterisation of the non-cooperative populations. Whenever the 1-coalitions are stable, there are no incentives for individuals to form pairs for the purpose of cooperation, as cooperation with another entity reduces the expected lifetimes of not extinct entities. Indeed, it can be proved that if the probability of being distressed is above a certain threshold, entities that are in survival will not take any risk to cooperate, as the probability of having to pair with a distressed entity is too high as compared with the benefits of future cooperation (the adverse selection risk is simply too high in this case). We give below the exact threshold for the probability:

Proposition 5.1 (The stable 1-coalitions). For any $c$ and $\frac{\gamma}{\alpha}$ fixed, there exists a critical probability:

$$
\pi_{1}=\max \left\{\frac{\gamma}{\alpha}+(1-c)^{2}, 1\right\}
$$

such that: if $\pi_{1}<1$, then for all $p>\pi_{1}$ the corresponding 1-coalition is stable and for all $p \leq \pi_{1}$ the corresponding 1-coalition is unstable. If $\pi_{1}=1$, then stable coalitions do not exist, regardless of the value of $p \in(0,1)$.

Proof. We impose the conditions Proposition 4.6 for stability to hold.

First of all we try to determine $n^{\text {out }}(1)$. Notice that for stable coalitions $0 \leq n^{\text {out }}(N) \leq N-1$, which in our case $(N=1)$ implies $n^{\text {out }}(1)=0$. For this to hold, we need: $h(1,2)=\frac{1}{2 \alpha c^{2}}\left(\frac{\gamma}{\alpha}+c^{2}\right)<\frac{1}{\alpha c}$ that is: $\frac{\gamma}{\alpha}+$ $(1-c)^{2}<1$. The conditions in Proposition 4.6 (i) are in this case only one: $h(0,1)>p h(1,2)+(1-p) h(0,2)$ where $h(0,1)=\frac{1}{\alpha c}$ and $h(0,2)=\frac{1}{2 \alpha c^{2}}\left(1+\frac{\gamma}{\alpha}+c^{2}\right)$. This condition is satisfied if and only if $\frac{\gamma}{\alpha}+(1-c)^{2}<p$. Condition (ii) in Proposition 4.6 is empty because $n^{\text {out }}=0$ and we have no inequalities to check. 
In Figure 3 we plot the boundary between stable and unstable 1-coalitions in the plane $\left(\frac{\gamma}{\alpha}, p\right)$, with $c$ being fixed at 0.8 . The non-cooperative populations are those having parameters in the white triangle and the cooperative populations have parameters in the grey area. The threshold $\pi_{1}$ is the line $\pi_{1}=p(x)=x+(1-c)^{2}$ that separates the white from the grey area. The blue curve is given by the function $p_{\infty}^{*}(x)=\frac{1-c}{1-x}$ and represents the limiting boundary between stable and unstable coalitions as $N \rightarrow \infty$ (in the next subsection, this limiting curve will be analysed in additional details). The area below the blue curve consists of coalitions that are optimally infinite. Consequently, the white areas that are situated below the blue curve are the strategically non-cooperative populations where the optimal decision consists in infinite cooperation. In other words, there the Nash equilibria are suboptimal: individual entities that are not distressed choose to not cooperate and hence they achieve an expected lifetime of $\frac{1}{\alpha c}$, but infinite cooperation would lead to an infinite lifetime.

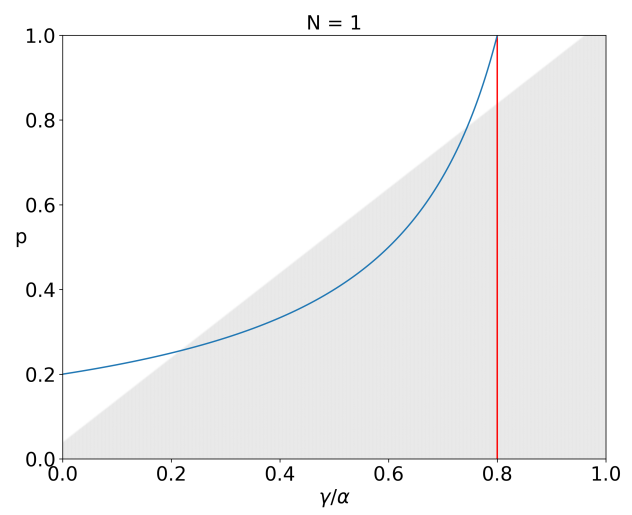

FIGURE 3. The repartition of the stable and unstable coalitions in the plane $\left(\frac{\gamma}{\alpha}, p\right)$ and for the sizes of the coalition $N=1$. The vertical red line is the level of the parameter $c$ that is fixed as $c=0.8$. Grey area: unstable coalitions; white area: stable coalitions.

5.2. Critical boundaries for stability. Critical probabilities are actually a general pattern:

Proposition 5.2. Suppose the parameters $\alpha, \gamma$ and $\delta$ fixed. For any $N$, there is a threshold probability $\pi_{N} \in[0,1]$, depending on the other parameters, such that for $p>\pi_{N}$ the coalitions are stable and for $p \leq \pi_{N}$ the coalitions are unstable.

Proof. Suppose that for any $p \in(0,1)$, no stable N-coalitions exist. The statement of the proposition is true in this case, with $\pi_{N}=1$.

Alternatively, suppose that there exists $p \in(0,1)$ such that the corresponding N-coalition is stable. Then, the conditions in (10) are verified (note that these do not depend on $p$ ). Also, the conditions in 9 ) are fulfilled for such $p$ and consequently

$$
\frac{h(n, N+1)-h(n, N)}{h(n, N+1)-h(n+1, N+1)}<1 \quad \text { for all } \in\left\{0, \ldots, n^{\text {out }}(N)\right\} .
$$


We denote $\pi_{N}:=\max _{n \in\left\{0, \ldots, n^{\text {out }}(N)\right\}} \frac{h(n, N+1)-h(n, N)}{h(n, N+1)-h(n+1, N+1)}$. It can be easily checked that with such $\pi_{N}$, an $\mathrm{N}$-coalition is stable if and only if $p>\pi_{N}$.

We now present a numerical study for stable and unstable regions in N-coalitions, using an algorithm based on Proposition 4.3

In Figure 4 , we analyse these regions in the $\left(\frac{\gamma}{\alpha}, p\right)$ plane for increasing values of $N$ as follows (from up left, to right and downward) $N=1, N=2, N=10 N=20$ and $N=100$. The parameters that are fixed for all pictures are $\alpha=0.5$ and $c=0.8$, while $p$ is varying between 0 and 1 and $\gamma$ is varying in the interval $[0,0.5]$ so that $\frac{\gamma}{\alpha} \in[0,1)$. As for the case $N=1$, the white area represents the stable coalitions and the unstable coalitions are given in the grey area. The blue curve represents the optimal probability threshold $\pi=\frac{1-c}{1-\frac{\gamma}{\alpha}}$ that was identified as the critical probability that separates optimal finite cooperation from optimal infinite cooperation (see Proposition 3.6 and expression (4)). In each subgraph of Figure 4 the critical probabilities $\left(\pi_{N}\right), N \geq 1$ are the boundaries separating the white from the grey areas. They are nondecreasing functions of $\gamma / \alpha$. Figure 4 shows that $\left(\pi_{N}\right)$ converge as $N$ increases to the asymptotic boundary $\pi$ and at $N=100$ it appears already very closed to it.

In our numerical investigation, we could observe such a convergence of the sequence $\left(\pi_{N}\right), N \geq 1$ to the value $\pi$ to occur very often. Convergence seems to break partially for large values of the parameter $c$ larger that 0.9 , where we could sometimes observe segments of the boundary $\pi_{N}$ stabilising above the level $\pi$ as $N$ increases. The (partial) convergence of $\pi_{N}$ towards $\pi$ implies that when imposing large coalition sizes (i.e., when the game $\Gamma$ starts with $N_{0}$ large), the differences between strategical versus optimal sizes of coalitions, tend to disappear. But this phenomenon alone gives no indication about the size of inefficiencies, in particular whenever the strategic size of coalitions is small. This question is analysed next.

5.3. Inefficiency zones of the Nash equilibria. In Figures 5, 6 and 7, we provide a detailed analysis of inefficiencies of strategic cooperation as compared to the optimal coalitions, in absence of intervention by a central planner or regulator. Optimal sizes of coalitions, denoted by $N^{*}$ were introduced and studied in Subection 3.2 In order to measure possible inefficiencies, we introduce $N^{\mathrm{min}}$, as being the size of the smallest stable coalition, that is: if $N=N^{\mathrm{min}}$ an $\mathrm{N}$-coalition is stable, and $N<N^{\mathrm{min}}$ implies an N-coalition is unstable. We set $N^{\mathrm{min}}=\infty$ whenever there are no stable coalitions. We shall name $N^{\mathrm{min}}$ strategic coalition size.

The size $N^{\text {min }}$ is the maximal size a group reaches within the strategic game $\Gamma=(\Gamma(N))_{N \geq 1}$, i.e., starting with a single entity, assumed non distressed. If stable coalitions exist, then the game evolves through larger and larger coalitions until it reaches a size where it is stable and then, the corresponding game never ends. This is indeed $N^{\mathrm{min}}$. Larger size games $\Gamma(N)$ with $N>N_{0}$ will not be played. However, it can be the case that the game $\Gamma$ stops before reaching the size $N^{\mathrm{min}}$, because of a smaller, unstable coalition becoming extinct, before getting the chance to enlarge. 

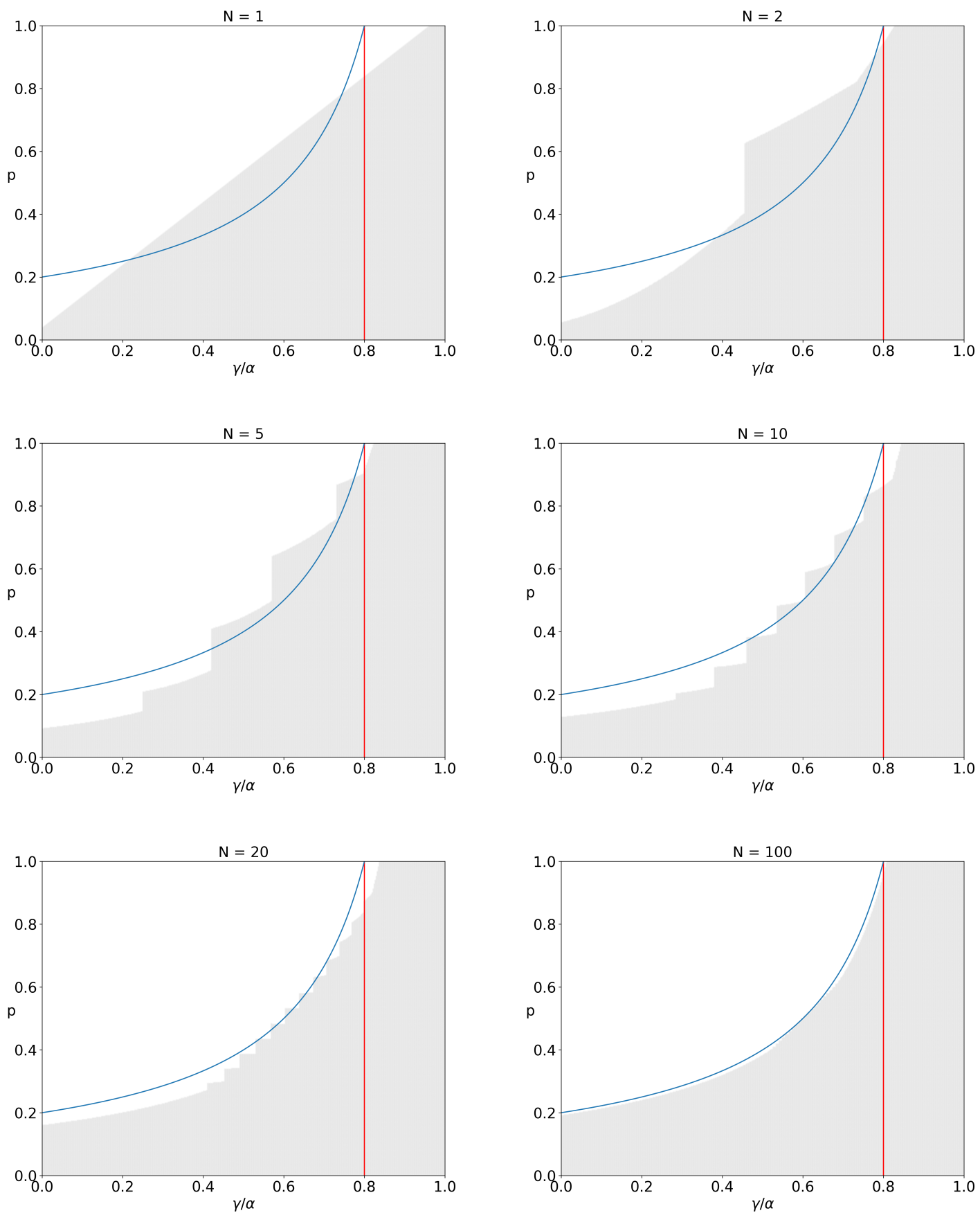

FIGURE 4. The repartition of the stable and unstable coalitions in the plane $\left(\frac{\gamma}{\alpha}, p\right)$ and for different sizes of the coalition $N$. The blue curve is the asymptotic frontier that delimitates the stable and unstable coalitions when $N \rightarrow \infty$, which is depending on $p$ and $\frac{\gamma}{\alpha}$. The vertical red line is the level of the parameter $c$ that is fixed as $c=0.8$. Grey area: unstable coalitions; white area: stable coalitions. 
In Figure 5, we display for different values of the parameter $c$ and in the plane $\left(\frac{\gamma}{\alpha}, c\right)$ the optimal $N^{*}$ versus the strategic $N^{\mathrm{min}}$ sizes of coalitions. Next, in Figure 6 expected lifetimes are measured, as corresponding to the optimal coalition size $N^{*}$ (left pictures) versus the strategic size $N^{\text {min }}$ (right pictures).

A last question is how far apart are the expected lifetimes corresponding to optimal cooperation from those corresponding to strategic cooperation? By answering this question, we obtain the clearest picture of the inefficiencies of the Nash equilibria. Figure 7 shows the expected lifetime differential between the optimal coalitions $N^{*}$ and the smallest stable coalitions $N^{\text {min }}$ (left pictures) and the same differential but in relative value (right pictures), displayed for different values of the parameters. We may observe that the higher inefficiencies are obtained around the optimal probability threshold $\pi$ separating the optimally finite from the optimally infinite coalitions. Inefficiencies can be infinite (the black areas) when $N^{\text {min }}<\infty$ while $N^{*}=\infty$. Also, relatively high inefficiencies, that can reach $60-70 \%$ in loss of expected lifetime are to be observed for the strategically individualist populations (having $N^{\mathrm{min}}=1$ ), where actual optimal groups would be larger, but finite, as of 20 or 50 members. The relative inefficiencies for these strategically individualist populations, are increasing as $\frac{\gamma}{\alpha}$ becomes close to $c$ and the probability $p$ increases to 1 , meaning high viability ratio and high risk adverse selection.

From the numerical analysis, we can draw some general conclusions regarding the size of cooperation, as follows:

- Strategic coalition sizes are less diverse in the space of parameters, as compared to optimal ones: for a majority of parameters' ranges, the strategic size $N^{\text {min }}$ is either 1 or infinite, and intermediary values are more rare. This means that in case of some external shocks of systemic nature, that would shift the values of the parameters of the model, the strategies move from one extreme to the other (i.e., form no cooperation to infinite cooperation). A central planner would instead choose more often intermediary range of coalition sizes (i.e., finite sizes with more than one element).

- Effect of the risk of adverse selection. Both optimal and strategic coalition sizes are decreasing with higher risk adverse selection (i.e., with higher $p$ ).

- Effect of the frequency of surplus creation given survival. The lower the value of $c$ is, the more frequent are non distressed entities able to detain a surplus. The analysis shows that ceteris paribus, the lower $\delta$, the lower $c$ and the more advantageous cooperation becomes. Indeed, in Figure 5, the areas coloured in black are wider with when $c$ lower, meaning that infinite cooperation prevails more often. This is true for both optimal and strategic cooperation.

- Effect of the viability ratio $\xi$. High viability ratio impacts positively the cooperation: the higher $\xi$, the higher the optimal and strategies sizes of coalitions (Figure 5) and the higher their corresponding expected lifetime (Figure 6). However, it seems that inefficiencies are also increasing with the viability ratio: in Figure 7, relative inefficiencies are increasing in $\xi$. 


\section{CONCLUDING REMARKS}

The aim of this paper was to investigate the extent to which cooperating groups are able to improve their survival chances, when survival depends on the availability of a resource, and assuming that this is fluctuating in time and through the population. Cooperation in this setting a mutual insurance mechanism. The question was answered both using Pareto optimality and a strategic game approach, based upon the notion of stable coalitions.

Introducing the notion of survival and the risk of extinction is new in the literature studying mutual insurance, as well as the continuous time framework, where we modeled the availability of the resource for each individual and point in time. Using only very few parameters, we were able to identify the elements that add value to cooperation, that are: limited adverse selection risk, high rate of surplus creation, or low rate of surplus depletion. The existence of thresholds for these parameters that separate infinite cooperation from finite cooperation was proved; different aspects of the inefficiencies of the Nash equilibria were analysed numerically. The highest inefficiencies were shown correspond to populations with parameter values situated close to the thresholds that separate infinite from finite optimal cooperation. These areas may also be interpreted as "fragility parameter zones", as small shifts in parameter values may bring cooperation from infinite to none.

Our model may be applied in several economic contexts involving risk sharing. The interbank liquidity markets are such an example of mutual insurance. Our model may help to understand how the liquidity in the system may evolve in absence of interventions by the central bank, and it displays the critical parameter values where the mutual insurance mechanism performs poorly (e.g., when there are no incentives to cooperate) and requires policy interventions. The model captures the phenomena of liquidity hoarding in times of high uncertainty about the credit quality of the counter-parties. This is a common feature in financial crises. Indeed, if the adverse selection parameter $p$ increases above a threshold, the populations in our model become individualistic; in the context of a financial system this may be interpreted as a disruption of the normal liquidity provision in times of high uncertainty. The stop in cooperation was shown to be abrupt: small variations of $p$ may provoke a population with infinite strategic cooperation to become individualistic (as seen in Figure 5, where for values of $p$ around the optimal threshold, small variations may have big impacts on the strategic sizes of cooperation). Consequently, by characterising these fragility zones, our model may help regulators by providing a guidance of when such disruptions are close to occur. Other applications, such as in insurance, or management of the surpluses of production at the level of a business group seem natural to consider and may bring in challenging new developments of our present model.

\section{A.APPENDICES}

A.1. Ressource availability with cooperation: definition and mathematical construction. This appendix contains the mathematical definition and construction of the process $\mathbf{L}^{(N)}$ that keeps track of the resource availability at each time $t \geq 0$ and for each entity $i \in\{1, \ldots, N\}$. 


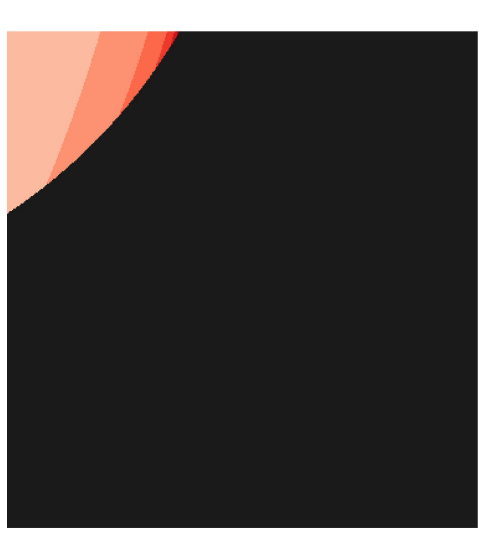

(a) Optimal coalition size for $c=0.4$

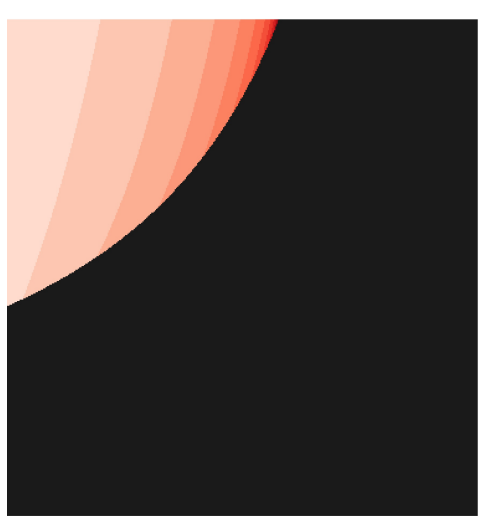

(c) Optimal coalition size for $c=0.6$

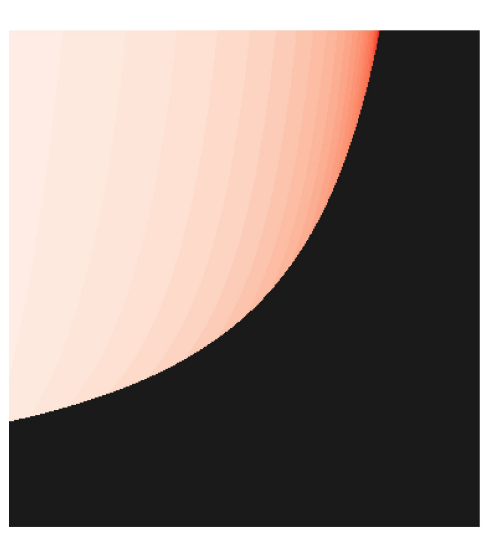

(e) Optimal coalition size for $c=0.8$
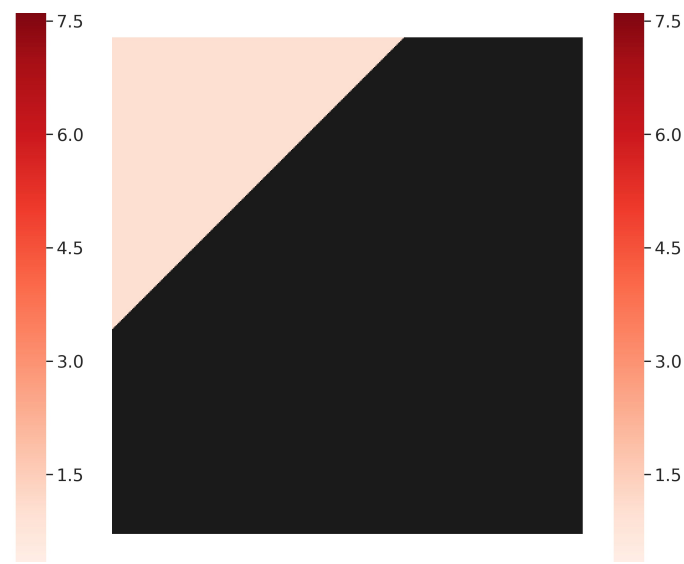

(b) Strategic coalition size for $c=0.4$
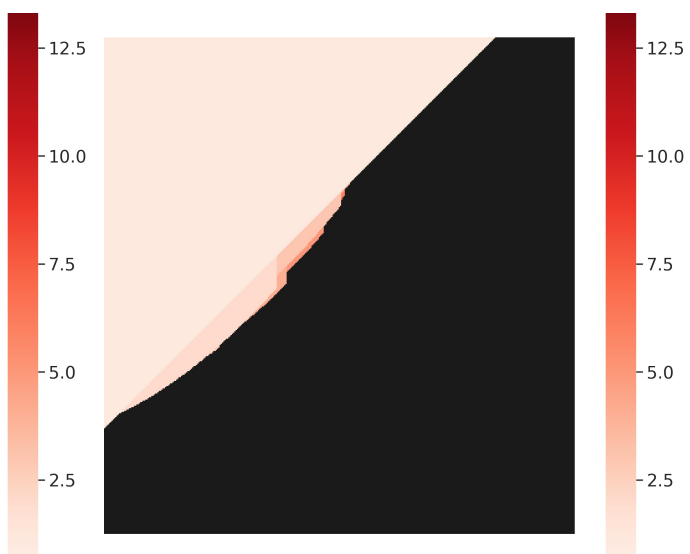

(d) Strategic coalition size for $c=0.6$

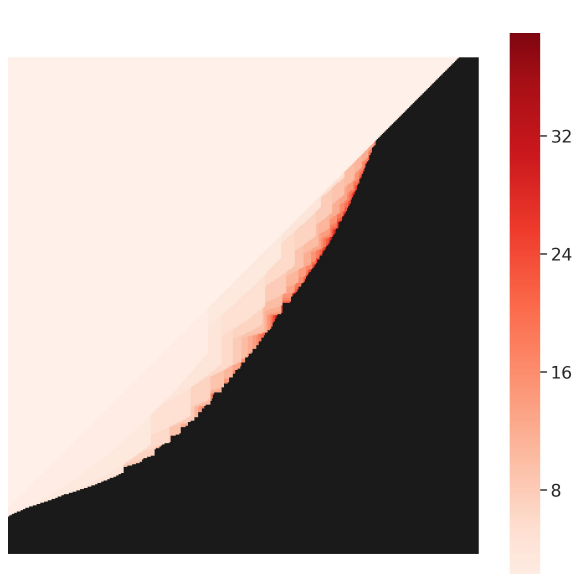

(f) Strategic coalition size for $c=0.8$

FIGURE 5. Optimal versus strategic sizes of coalitions in the plane $\left(\frac{\gamma}{\alpha}, p\right)$ and for different values of the parameter $c$. The black colour represents infinite sizes, the other colours are in the legend. 


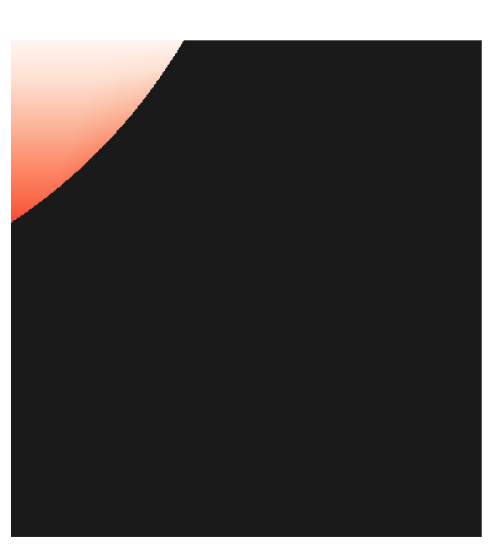

(a) Optimal expected lifetime for $c=0.4$
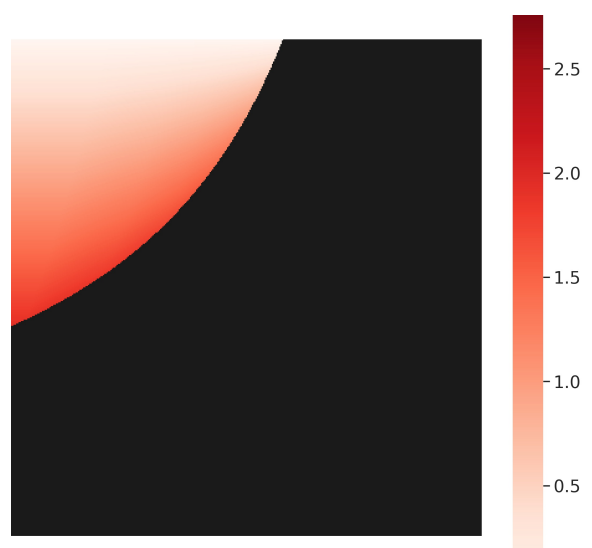

(c) Optimal expected lifetime for $c=0.6$

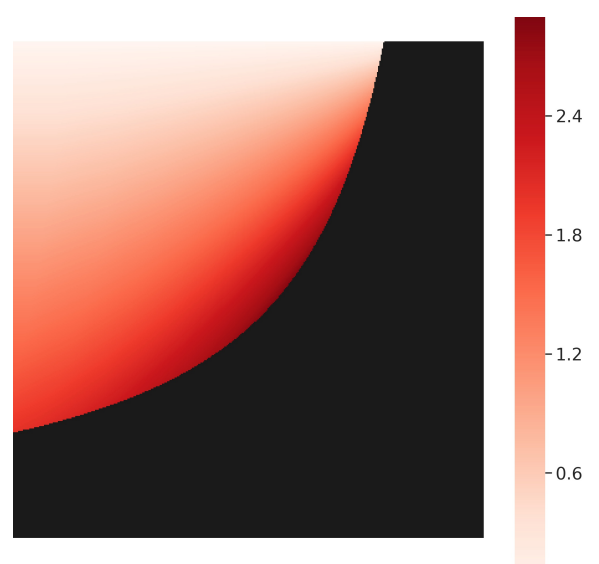

(e) Optimal expected lifetime for $c=0.8$

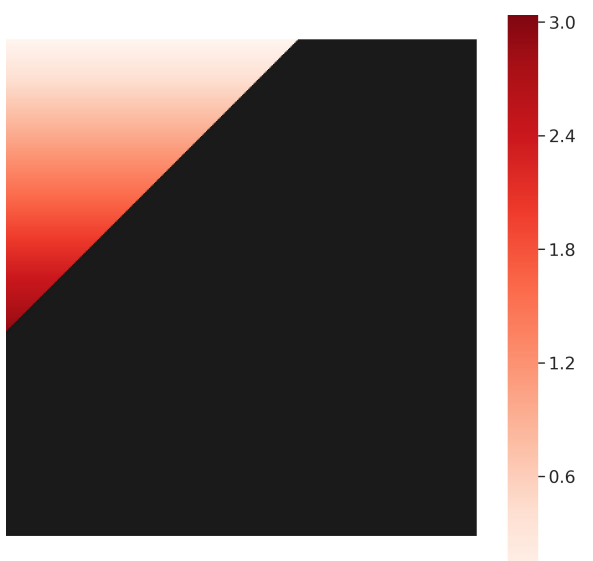

(b) Strategic expected lifetime for $c=0.4$

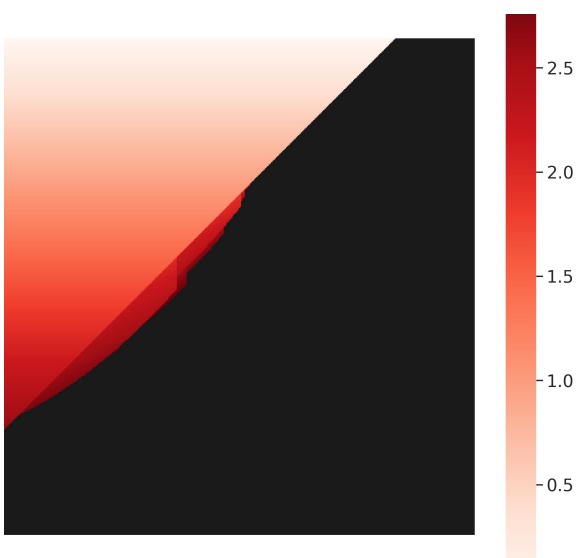

(d) Strategic expected lifetime for $c=0.6$

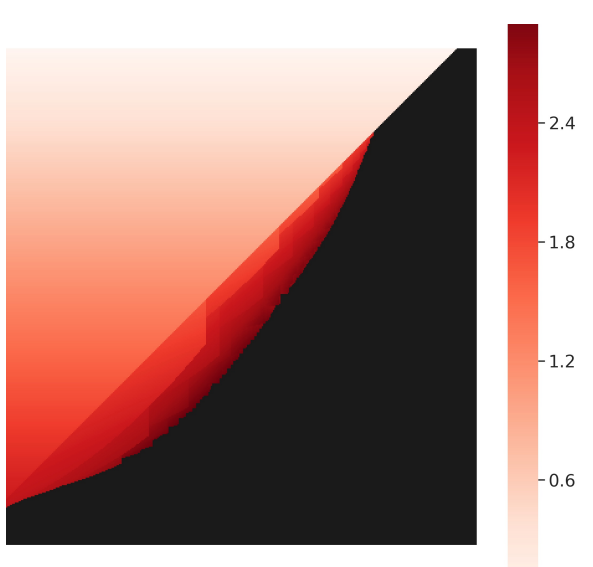

(f) Strategic expected lifetime for $c=0.8$

FIGURE 6. Expected lifetimes corresponding to optimal coalition sizes $N^{*}$, versus strategic coalition sizes $N^{\min }$ in the plane $\left(\frac{\gamma}{\alpha}, p\right)$ and for different values of the parameter $c$. The black colour represents infinite lifetimes, the other colours are in the legend. 

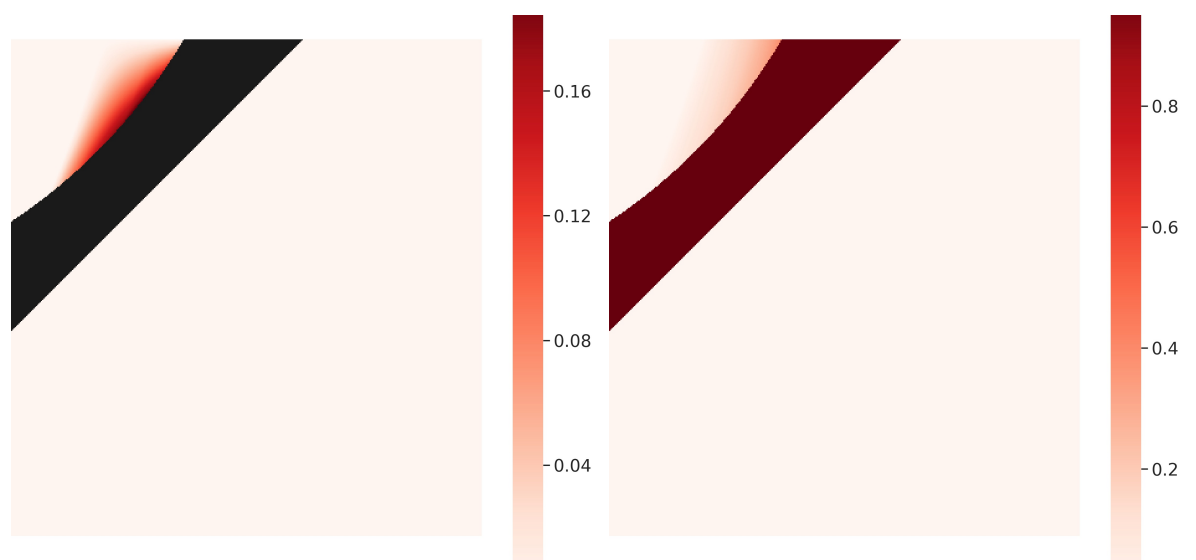

(a) Absolute loss, $c=0.4$.

(b) Relative loss, $c=0.4$.
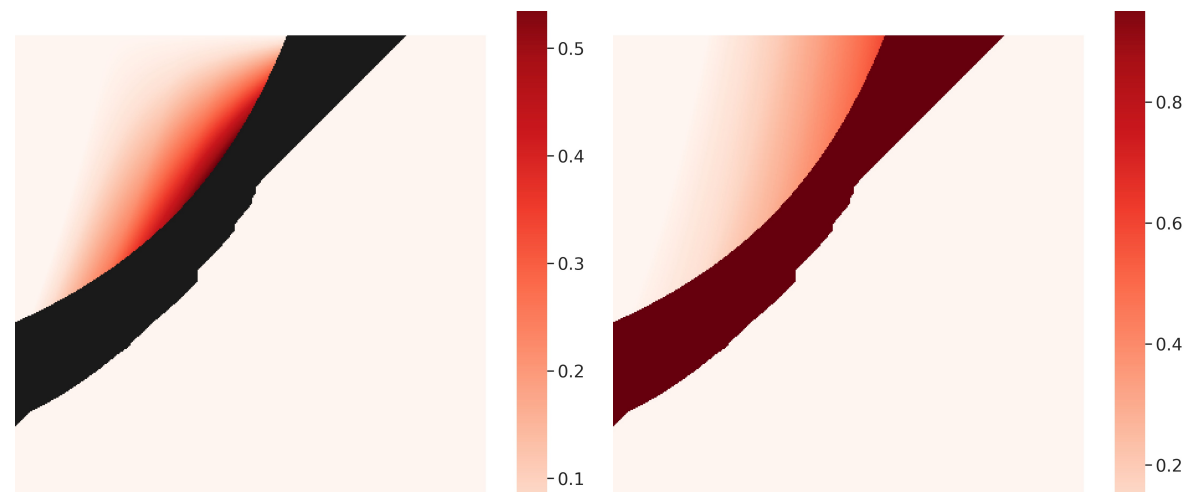

(c) Absolute loss, $c=0.6$.

(d) Relative loss, $c=0.6$.

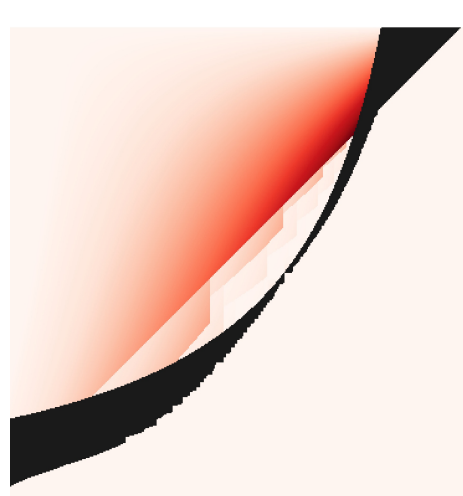

(e) Absolute loss, $c=0.8$.
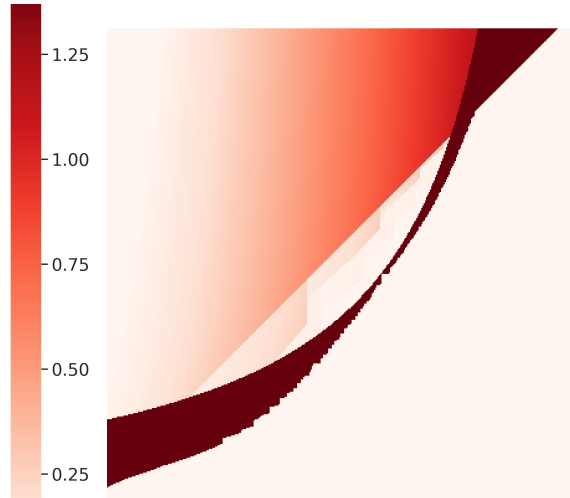

(f) Relative loss, $c=0.8$.

FIGURE 7. Losses of strategic cooperation of size $N^{\min }$ as compared to the optimal size $N^{*}$, measured in expected lifetime differential, displayed in the plane $\left(\frac{\gamma}{\alpha}, p\right)$ for different values of the parameter $c$. The black colour represents infinite absolute losses; we normalise these to 1 to obtain relative losses. The other relative losses are given by absolute losses divided by expected lifetime for optimal coalitions. 
First, we introduce the main objects involved: the interaction times, i.e., when cooperation occurs, the evolution of the state process $\mathbf{L}^{(N)}$ between these interaction times, and the concrete effect of cooperation when it occurs.

The interaction times are defined as a sequence of stopping times $(\theta(n), n \geq 1)$, representing the ordered times when interaction occurs within the group. As explained already, interaction consists in one distressed entity being rescued by another entity, member of the coalition, having a surplus. We assume that these are the only interactions between the components of the process $\mathbf{L}^{(N)}$ and in between these times, i.e., for $t \in[\theta(n), \theta(n+1))$, the components of the vector process $\mathbf{L}^{(N)}$ are evolving independently one from another.

Consequently, the law of the state process between any two successive transaction times, is described by a stochastic process $\boldsymbol{\ell}=\left(\ell_{t}(1), \cdots, \ell_{t}(N)\right)_{t \geq 0}$ taking values on $\{-1,0,+1\}^{N}$ with components being independent Markov chains, with the evolution of any $\ell(k)$, component $k$ of $\ell$, given by the transition matrix $\mathbf{Q}^{(1)}$ in $(1)$. We shall use the notation $\boldsymbol{\ell}^{\mathbf{x}}$ for the process $\boldsymbol{\ell}$ satisfying the initial condition $\boldsymbol{\ell}_{0}=\mathbf{x}$, for some $\mathbf{x} \in\{-1,0,+1\}^{N}$.

The effect of cooperation is to reverse the state of distress of an entity, whenever surplus is available (i.e. whenever there are entities in the surplus state within the coalition). Therefore, the process $\ell$ will be reflected back into the set $I$ whenever it exits. The successive exit times from $I$ are precisely the transaction times $(\theta(n))$ mentioned above. It remains to define the reflection functions, which specify the state of the process $\mathbf{L}^{(N)}$ after transactions occur. This will be done in Definition A.3 below.

Now, some additional notation:

Notation A.1. We introduce the following functions, defined on $\{-1,0,+1\}^{N}$ with values in $\{0, \cdots, N\}$ :

$\eta^{-}(\mathbf{x}):=|\{i: x(i)=-1\}|$,i.e. the number of distressed entities, when the state of the coalition is $\mathbf{x}$,

$\eta^{+}(\mathbf{x}):=|\{i: x(i)=+1\}|$,i.e. the number of entities with a surplus, when the state of the coalition is $\mathbf{x}$, where we denote by $|A|$ the cardinality of the set $A$, for A a countable set.

Notation A.2. We partition the set $E:=\{-1,0,+1\}^{N} \backslash I$ in two disjoint sets as follows:

(i) The states where there are at least as many entities with a surplus as entities in distress, with at least one entity in distress:

$$
E_{+}:=\left\{\mathbf{x} \in\{-1,0,+1\}^{N} \mid \eta^{+}(\mathbf{x}) \geq \eta^{-}(\mathbf{x}) \geq 1\right\} .
$$

(ii) The states where there are more distressed entities than entities with a surplus, with at least one entity with surplus:

$$
E_{-}:=\left\{\mathbf{x} \in\{-1,0,+1\}^{N} \mid \eta^{-}(\mathbf{x})>\eta^{+}(\mathbf{x}) \geq 1\right\} .
$$

Definition A.3 (The reflecting functions). We define a family of functions $\left(\mathcal{R}_{n}\right)_{n \geq 0}$ as follows. For any $n \geq 1$, the function $\mathcal{R}_{n}(\mathbf{x})=\left(\mathcal{R}_{n}(\mathbf{x})(1), \cdots, \mathcal{R}_{n}(\mathbf{x})(N)\right)$

$$
\mathcal{R}_{n}: E \times \Omega \rightarrow I
$$


associates with an element $\mathbf{x} \in E$ an I valued random variable as follows:

(i) If $\mathrm{x} \in E_{+}$, then

$$
\mathcal{R}_{n}(\mathbf{x})(k)=B_{n, k}, \quad k \in\{1, \cdots, N\},
$$

where $\left(B_{n, k}\right)_{k \in\{1, \cdots, N\}, n \geq 0}$ is a family of independent and identically distributed random variable Bernoulli distributed with parameter $(1-c)$, where we recall that $c=\frac{\delta}{\gamma+\delta}$.

(ii) If $\mathbf{x} \in E_{-}$, then

$$
\mathcal{R}_{n}(\mathbf{x})(k)= \begin{cases}0 & \text { for } k \in\{i: x(i)=+1\} \cup C_{n}(\mathbf{x}), \\ \mathbf{x}(k) & \text { for } k \in\{i: x(i)=0\} \cup\{i: x(i)=-1\} \backslash C_{n}(\mathbf{x}),\end{cases}
$$

where for any $\mathbf{x} \in E_{-},\left(C_{n}(\mathbf{x})\right)_{n \geq 0}$ is a family of independent random variables, where $C_{n}(\mathbf{x})$ is uniform on the set of all subsets of $\{i: x(i)=-1\}$ having precisely $\eta^{+}(\mathbf{x})$ elements.

The interpretation of the reflection functions is in line with our previous description of cooperation. In $E_{+}$, there are more entities with surplus than distressed entities. Therefore transfers of the resource may occur and allow all distressed entities to recover. The reflection function applied to elements in $E_{+}$attributes only values 0 and +1 to all entities, so that no entity will be in distress. Importantly, the values are chosen so that Condition 2 is verified (as proved in Lemma A.5 below).

When $\mathbf{x} \in E_{-}$, there are more distressed entities than entities with a surplus. An entity with a surplus will randomly choose a distressed entity and a transfer of the resource takes place; after the transfer, both entities reach the neutral state 0 . The set of all distressed entities that will recover is given by $C_{n}(\mathbf{x})$, with cardinal $\eta^{+}(\mathbf{x})$, containing randomly selected elements among the set of all distressed entities. We observe that not all distressed entities can be rescued in $E_{-}$. There will be precisely $\eta^{-}(\mathbf{x})-\eta^{+}(\mathbf{x}) \geq 1$ distressed entities that cannot recover and they remain in the state -1 ; these are the elements of the set $\{i: x(i)=-1\} \backslash C_{n}(\mathbf{x})$. The neutral entities (that are in state 0 ) will keep their state 0 unchanged when applying the reflecting function.

Definition A.4 (The resource availability process). The resource availability process of the $\mathrm{N}$-coalition is a stochastic process denoted $\mathbf{L}^{(N)}$ taking values in the set:

$$
I=\left\{\mathbf{x} \in\{-1,0,+1\}^{N} \mid \min \left\{\eta^{-}(\mathbf{x}), \eta^{+}(\mathbf{x})\right\}=0\right\},
$$

with:

$$
\mathbf{x}_{0}=\mathcal{R}_{0}\left(\ell_{0}\right) \quad \text { for an initial state } \ell_{0} \in\{-1,0,+1\}^{N} .
$$

We denote $\theta(0)=0$ and for $n \in \mathbb{N}$, we define $\mathbf{L}^{(N)}$ recursively:

$$
\mathbf{L}_{t}^{(N)}=\ell_{t-\theta(n)}^{\mathbf{x}_{n}} \text { for } t \in[\theta(n), \theta(n+1)),
$$


where:

$$
\begin{aligned}
\theta(n+1) & :=\theta(n)+\inf \left\{t \geq 0 \mid \ell_{t}^{\mathbf{x}_{n}} \in E\right\}, n \geq 0, \text { and } \\
\mathbf{x}_{n+1} & :=\mathcal{R}_{n+1}\left(\ell_{\theta(n+1)}^{\mathbf{x}_{n}}\right) .
\end{aligned}
$$

Above, the quantity $\mathcal{R}_{n+1}\left(\ell_{\theta(n+1)}^{\mathbf{x}_{n}}\right)$ defines the state in which the process $\mathbf{L}^{(N)}$ finds itself after the transaction $n+1$, taking place at time $\theta(n+1)$. The random functions $\left(\mathcal{R}_{n}\right)_{n \geq 0}$ each associates to a state in E a state in I (see Definition A.3).

Note that there is a sequence of reflecting functions, and not only one reflecting function, and we used a unique reflecting function $\mathcal{R}_{n}$ per transaction $\theta(n)$. This way, there will be independence of the state process of the previously visited states, by using an independent random variable at each time to allocate the resource among the coalition members. Nevertheless, we emphasise that we only need the distribution of the entities after a transaction takes place, as will be shown in the next sections, so that we could also have used a single reflecting function in our definition above, without affecting the validity of our analysis.

Also, we can notice that the state space of the process $\mathbf{L}^{(N)}$ satisfies Condition 1 . We now verify that Condition 2 is satisfied as well.

Lemma A.5. The process $\mathbf{L}^{(N)}$ from Definition A.4 satisfies Condition 2, namely, for all $t \geq 0$ and for all $A \in\{-1,0,+1\}^{N}$ :

$$
\mathbb{P}\left(\mathbf{L}_{t}^{(N)} \in A \mid \eta^{-}\left(\mathbf{L}_{t}^{(N)}\right)=0\right)=\overline{\boldsymbol{\mu}}^{N}(A)
$$

and with $\overline{\boldsymbol{\mu}}^{N}$ the product measure associated with $\overline{\boldsymbol{\mu}}$ defined in (2).

Proof.

By definition, $\eta^{-}\left(\mathbf{L}_{t}^{(N)}\right)=0$ means there are no distressed entities, that is:

$$
\left\{\eta^{-}\left(\mathbf{L}_{t}^{(N)}\right)=0\right\}=\left\{\mathbf{L}_{t}^{(N)} \in\{0,+1\}^{N}\right\}
$$

Obviously, if $A \notin\{0,+1\}^{N}$, then $\mathbb{P}\left(\mathbf{L}_{t}^{(N)} \in A \mid \eta^{-}\left(\mathbf{L}_{t}^{(N)}\right)=0\right)=0=\overline{\boldsymbol{\mu}}^{N}(A)$ by definition of $\overline{\boldsymbol{\mu}}^{N}$.

Now, suppose $A \in\{0,+1\}^{N}$. We remark that $\{0,+1\}^{N} \subset I$. We consider $t$ fixed, with $t \in\left[\theta_{n}, \theta_{n+1}\right]$ for some $n$, using the notation from Definition A.4. We write the event $\left\{\mathbf{L}_{t}^{(N)} \in\{0,+1\}^{N}\right\}$ as the union of two disjoint events:

$$
B:=\left\{\mathbf{L}_{t}^{(N)} \in\{0,+1\}^{N}\right\}=B_{1} \cup B_{2},
$$

where $B_{1}=\left\{\boldsymbol{\ell}_{t-\theta_{n}}^{\mathbf{x}_{n}} \in\{0,+1\}^{N}\right\}$ and $B_{2}=\left\{\boldsymbol{\ell}_{t-\theta_{n}}^{\mathbf{x}_{n}} \notin I\right\} \cap\left\{\mathcal{R}\left(\ell_{t-\theta_{n}}^{\mathbf{x}_{n}}\right) \in\{0,+1\}^{N}\right\}$. The result will follow from the analysis below:

Suppose that $\ell_{t-\theta_{n}}^{\mathbf{x}_{n}} \in I$. The law of $\ell_{t}^{\mathbf{x}_{n}}$ conditionally on $\left\{\boldsymbol{\ell}_{t}^{\mathbf{x}_{n}} \in\{0,+1\}^{N}\right\}$ is $\overline{\boldsymbol{\mu}}^{N}$. Indeed, all components of $\boldsymbol{\ell}$ are independent and $\overline{\boldsymbol{\mu}}^{N}$ is the product measure of $\overline{\boldsymbol{\mu}}$ defined in 22. Conditionally on $B_{1}$, $\mathbf{L}_{t}^{(N)}=\ell_{t-\theta_{n}}^{\mathbf{x}_{n}}$ therefore the law of $\mathbf{L}_{t}^{(N)}$ is $(\overline{\boldsymbol{\mu}})^{N}$, conditionally on $B_{1}$. 
Suppose that $\ell_{t-\theta_{n}}^{\mathbf{x}_{n}} \notin I$. Then, $\mathbf{L}_{t}^{(N)}=\mathcal{R}\left(\ell_{t-\theta_{n}}^{\mathbf{x}_{n}}\right) \in A$, by definition of the process $\mathbf{L}^{(N)}$. In this case, $t=\theta_{n+1}$. By Definition A.3 for the process $\boldsymbol{\ell}$ to be reflected in a point belonging to $A \subset\{0,+1\}^{N}$, the reflection function necessarily is applied to the region $E_{+}$so that we have all $\mathbf{L}_{t}^{(N)}(k)$ independent and identically distributed random variables Bernoulli with parameter $(1-c)$. This is to say $\overline{\boldsymbol{\mu}}^{N}$ is also the law of $\mathbf{L}_{t}^{(N)}$ conditionally on $B_{2}$.

Therefore:

$$
\begin{aligned}
\mathbb{P}\left(\mathbf{L}_{t}^{(N)} \in A \mid B\right) & =\mathbb{P}\left(\mathbf{L}_{t}^{(N)} \in A \mid B_{1}\right) \cdot \mathbb{P}\left(B_{1} \mid B\right)+\mathbb{P}\left(\mathbf{L}_{t}^{(N)} \in A \mid B_{2}\right) \cdot \mathbb{P}\left(B_{2} \mid B\right) \\
& =\overline{\boldsymbol{\mu}}^{N}(A) \cdot\left(\mathbb{P}\left(A_{1} \mid B\right)+\mathbb{P}\left(B_{2} \mid B\right)\right)=\overline{\boldsymbol{\mu}}^{(N)}(A) .
\end{aligned}
$$

A.2. Proof of Theorem 3.2. For simplicity we use the notations $Y$, instead of $Y^{(N)}$. We denote by $\left\{P_{t}, t \geq 0\right\}$ the semigroup associated with the transition matrix $\mathbf{Q}^{(N)}$. This means that $P_{t}$ is characterised by an infinitesimal generator:

$$
A h(n)= \begin{cases}{[\varphi(1)-\varphi(0)] \alpha N c^{N}} & \text { for } n=0, \\ {[\varphi(n+1)-\varphi(n)] \alpha(N-n)+[\varphi(n-1)-\varphi(n)] \gamma(N-n)} & \text { for } n=1, . ., N\end{cases}
$$

for any function $\varphi$ bounded and continuous, defined as $\varphi:\{0, \cdots, N\} \rightarrow \mathbb{R}$. The semigroup $P_{t}$ and its generator are linked by:

$$
P_{t} \varphi-\varphi=\int_{0}^{t} P_{s} A \varphi d s
$$

We denote by $\mathcal{F}_{t}^{Y}:=\sigma\left(Y_{s}, s \leq t\right)$, the information about the distress level up to time $t$. We need to prove that for any bounded continuous function $\varphi$ we have:

$$
\mathbb{E}\left[\varphi\left(Y_{t}\right) \mid \mathcal{F}_{s}^{Y}\right]=\mathbb{E}\left[\varphi\left(Y_{t}\right) \mid Y_{s}\right]=P_{t-s} \varphi\left(Y_{s}\right),
$$

or, alternatively in view of the relation (13):

$$
\mathbb{E}\left[\varphi\left(Y_{t}\right)-\varphi\left(Y_{s}\right) \mid \mathcal{F}_{s}^{Y}\right]=\int_{s}^{t} \mathbb{E}\left[\varphi\left(Y_{u}\right) \mid Y_{s}\right] \mathrm{d} u, \quad s \leq t .
$$

where $\mathbb{E}_{x}$ denotes the expectation under $\mathbb{P}$ starting from $x$. We use the technique of localisation to show that the relation (14) holds for any $s \leq t$, see for eg. [16], page 36 .

We consider the $\mathrm{N}$-dimensional Markov chain with independent components $\ell=(\ell(1), \cdots, \ell(N))$, as introduced in the previous section, i.e., $\ell$ is the canonical realisation of the semigroup $P_{t}^{\ell}$ characterised by the following infinitesimal generator:

$$
A^{\ell} f(\mathbf{x})=\sum_{i: x(i)=0}\left\{\left[f\left(\mathbf{x}+\mathbf{e}_{i}\right)-f(\mathbf{x})\right] \gamma+\left[f\left(\mathbf{x}-\mathbf{e}_{i}\right)-f(\mathbf{x})\right] \alpha\right\}+\sum_{i: x(i)=+1}\left[f\left(\mathbf{x}-\mathbf{e}_{i}\right)-f(\mathbf{x})\right] \delta,
$$


with $f:\{-1,0,+1\}^{N} \rightarrow \mathbb{R}, f$ continuous bounded, $\mathbf{e}_{i}=(0, \ldots, 1, \ldots, 0)$, where 1 is the $i$ component. The positive constants $\alpha, \gamma$ and $\delta$ are the ones from (1).

In what follows we use Notation A.1 and A.2, and the additional notation:

$$
\eta^{0}(\xi):=|\{i: x(i)=0\}|,
$$

so that $\eta^{-}(\mathbf{x})+\eta^{0}(\mathbf{x})+\eta^{+}(\mathbf{x})=N$ for any $\mathbf{x} \in\{-1,0,+1\}^{N}$.

Let us fix a state $\mathrm{x} \in I$; the neighbouring states (i.e., reachable from $\mathrm{x}$ after one transition of $\ell$ ) are either in $I$ or, when the process exits form $I$, at the boundary of $E$, defined as:

$$
\partial E:=\left\{\mathbf{x} \in\{-1,0,+1\}^{N} \mid \min \left\{\eta^{+}(\mathbf{x}), \eta^{-}(\mathbf{x})\right\}=1\right\} .
$$

Indeed, the process $\ell$ has independent components, so that it can evolve only by one component at a time. Additionally, we denote by

$$
\partial E_{+}:=\partial E \cap E_{+} \quad \text { and } \quad \partial E_{-}:=\partial E \cap E_{-} .
$$

We introduce the function:

$$
g(\mathbf{x}):=\max \left\{\eta^{-}(\mathbf{x})-\eta^{+}(\mathbf{x}), 0\right\} .
$$

We notice that for $\mathbf{x} \in I$ the function $g$ counts the number of distressed entities, that is:

$$
g(\mathbf{x})=\eta^{-}(\mathbf{x}) \text { for all } \mathbf{x} \in I,
$$

and at the boundary of $E$ the function $g$ removes one unit from the set of distressed entities:

$$
g(\mathbf{x})=\eta^{-}(\mathbf{x})-1 \text { for all } \mathbf{x} \in \partial E .
$$

For a fixed state $\mathbf{x} \in I$, let us detail the values taken by $g$ for the neighbouring states:

(i) If $\eta^{-}(\mathbf{x})=0$ and $\eta^{+}(\mathbf{x})=0$ (hence $\eta^{0}(\mathbf{x})=N$, i.e., $\mathbf{x}=\mathbf{0}$ ), then for $i=1, \cdots, N$, the neighbouring states are:

$$
\begin{aligned}
& \mathbf{x}+\mathbf{e}_{i} \in I \text { and } g\left(\mathbf{x}+\mathbf{e}_{i}\right)=\eta^{-}\left(\mathbf{x}+\mathbf{e}_{i}\right)=0, \\
& \mathbf{x}-\mathbf{e}_{i} \in I \text { and } g\left(\mathbf{x}-\mathbf{e}_{i}\right)=\eta^{-}\left(\mathbf{x}-\mathbf{e}_{i}\right)=\eta^{-}(\mathbf{x})+1=1 .
\end{aligned}
$$

(ii) If $\eta^{-}(\mathbf{x})=0$ and $\eta^{+}(\mathbf{x})>0$, then the neighbouring states are:

(ii.1) For $i \in\{k \mid x(k)=0\}$ :

$\mathbf{x}+\mathbf{e}_{i} \in I$ and $g\left(\mathbf{x}+\mathbf{e}_{i}\right)=\eta^{-}\left(\mathbf{x}+\mathbf{e}_{i}\right)=0$,

$\mathbf{x}-\mathbf{e}_{i} \in \partial E_{+}$and $g\left(\mathbf{x}-\mathbf{e}_{i}\right)=\eta^{-}\left(\mathbf{x}-\mathbf{e}_{i}\right)-1=\eta^{-}(\mathbf{x})+1-1=0$.

(ii.2) For $i \in\{k \mid x(k)=+1\}$ :

$\mathbf{x}-\mathbf{e}_{i} \in I$ and $g\left(\mathbf{x}-\mathbf{e}_{i}\right)=\eta^{-}\left(\mathbf{x}-\mathbf{e}_{i}\right)=\eta^{-}(\mathbf{x})=0$.

(iii) If $\eta^{-}(\mathbf{x}) \in\{1, \cdots, N-1\}$, then (by definition of the set $I$ ) $\eta^{+}(\mathbf{x})=0$, the neighbouring states are:

(iii.1) For $i \in\{k \mid x(k)=0\} \mathbf{x}+\mathbf{e}_{i} \in \partial E_{+}$and $g\left(\mathbf{x}+\mathbf{e}_{i}\right)=\eta^{-}\left(\mathbf{x}+\mathbf{e}_{i}\right)-1=\eta^{-}(\mathbf{x})-1$, $\mathbf{x}+\mathbf{e}_{i} \in \partial E_{-}$and $g\left(\mathbf{x}+\mathbf{e}_{i}\right)=\eta^{-}\left(\mathbf{x}+\mathbf{e}_{i}\right)-1=\eta^{-}(\mathbf{x})-1$, 


$$
\mathbf{x}-\mathbf{e}_{i} \in I \text { and } g\left(\mathbf{x}-\mathbf{e}_{i}\right)=\eta^{-}\left(\mathbf{x}-\mathbf{e}_{i}\right)=\eta^{-}(\mathbf{x})+1 .
$$

(iii.2) For $i \in\{k \mid x(k)=-1\}$ : no neighbouring states, as these states are absorbing.

(iv) If $\eta^{-}(\mathbf{x})=N$, then $\mathbf{x}=(-1, \cdots,-1) \in I$, i.e., the absorbing state for $\boldsymbol{\ell}$, meaning that it has no neighbouring states.

Therefore, for $\mathbf{x} \in I$ we have:

$$
\begin{aligned}
A^{\ell} \varphi \circ g(\mathbf{x})= & \mathbf{1}_{\left\{\eta^{0}(\mathbf{x})=N\right\}} \cdot[\varphi(1)-\varphi(0)] \alpha N \\
& +\mathbf{1}_{\left\{\eta^{-}(\mathbf{x})>0\right\}} \cdot \eta^{0}(\mathbf{x})\left\{\left[\varphi\left(\eta^{-}(\mathbf{x})-1\right)-\varphi\left(\eta^{-}(\mathbf{x})\right)\right] \gamma+\left[\varphi\left(\eta^{-}(\mathbf{x})+1\right)-\varphi\left(\eta^{-}(\mathbf{x})\right)\right] \alpha\right\} \\
= & \mathbf{1}_{\{\mathbf{x}=\mathbf{0}\}}[\varphi(1)-\varphi(0)] \alpha N \\
& +\sum_{n=1}^{N} \mathbf{1}_{\left\{\eta^{-}(\mathbf{x})=n\right\}} \cdot(N-n)\{[\varphi(n-1)-\varphi(n)] \gamma+[\varphi(n+1)-\varphi(n)] \alpha\} .
\end{aligned}
$$

Above, we have used the fact that: if $\mathbf{x} \in I$ and $\eta^{-}(\mathbf{x})=n>0$, then $\eta^{+}(\mathbf{x})=0$ and hence $\eta^{0}(\mathbf{x})=N-n$.

We define the first exit time of $\ell$ from $I$ as

$$
T_{1}(\omega):=\inf \left\{t \geq 0 \mid \ell_{t}(\omega) \notin I\right\},
$$

and we introduce the shift operator $\tau(t), t \geq 0$ defined as:

$$
\ell_{s}(\tau(t, \omega))=\ell_{t+s}(\omega) .
$$

(see Revuz and Yor [27] page 36 for more details). It follows that $T_{1} \circ \tau(s)=\inf \left\{t \geq s \mid \ell_{t}(\omega) \notin I\right\}$. Also, we define recursively $T_{n}=T_{1} \circ \tau\left(T_{n-1}\right)$ for $n>1$. It follows from the definition of $Y$ and the relations (17)-(18) that the following relation holds:

$$
Y_{t \wedge T_{1}}=g\left(\ell_{t \wedge T_{1}}\right)
$$

We first show the property (14) up to the first exit time from $I$, which is a stopping time for $\left(\mathcal{F}_{t}^{\ell}\right)$ (the filtration of the process $\ell$ ), but it is not a stopping time in $\left(\mathcal{F}_{t}^{Y}\right)$, the filtration of the process $Y$. We consider $\varphi$ as above and denote

$$
f:=\varphi \circ g,
$$

with $g$ as in 16 . We obtain:

$$
\begin{aligned}
\mathbb{E} & {\left[\varphi\left(Y_{t \wedge\left(T_{1} \circ \tau(s)\right)}\right)-\varphi\left(Y_{s}\right) \mid \mathcal{F}_{s}^{Y}\right]=\mathbb{E}\left[f\left(\boldsymbol{\ell}_{t \wedge\left(T_{1} \circ \tau(s)\right)}\right)-f\left(\boldsymbol{\ell}_{s}\right) \mid \mathcal{F}_{s}^{Y}\right] } \\
& =\mathbb{E}\left[\mathbb{E}\left[f\left(\boldsymbol{\ell}_{t \wedge\left(T_{1} \circ \tau(s)\right)}\right)-f\left(\boldsymbol{\ell}_{s}\right) \mid \mathcal{F}_{s}^{\ell}\right] \mid \mathcal{F}_{s}^{Y}\right]=\mathbb{E}\left[\mathbb{E}\left[\int_{s}^{t \wedge\left(T_{1} \circ \tau(s)\right)} A^{\ell} f\left(\boldsymbol{\ell}_{u}\right) d u \mid \boldsymbol{\ell}_{s}\right] \mid \mathcal{F}_{s}^{Y}\right] \\
& =\mathbb{E}\left[\int_{s}^{t} \mathbb{E}\left[\mathbf{1}_{\left\{T_{1} \circ \theta_{s}>u\right\}} \cdot A^{\ell} f\left(\boldsymbol{\ell}_{u}\right) \mid \mathcal{F}_{u}^{Y}\right] d u \mid \mathcal{F}_{s}^{Y}\right] .
\end{aligned}
$$


We now compute the inner expectations above. If $T_{1}>u$ then $\ell_{u} \in I$. Consequently, using (17), we have

$$
\mathbf{1}_{\left\{T_{1}>u\right\}} \cdot f\left(\boldsymbol{\ell}_{u}\right)=\mathbf{1}_{\left\{T_{1}>u\right\}} \cdot \varphi\left(\eta^{-}\left(\boldsymbol{\ell}_{u}\right)\right),
$$

and using (19) we get the following.

Firstly, we suppose $Y_{u}=0$, this is equivalent to $\eta^{-}\left(\ell_{u}\right)=0$. We obtain

$$
\begin{aligned}
\mathbf{1}_{\left\{Y_{u}=0\right\}} & \cdot \mathbb{E}\left[\mathbf{1}_{\left\{T_{1} \circ \tau(s)>u\right\}} \cdot A^{\ell} f\left(\boldsymbol{\ell}_{u}\right) \mid \mathcal{F}_{u}^{Y}\right]=\mathbf{1}_{\left\{Y_{u}=0\right\}} \cdot \mathbb{E}\left[\mathbf{1}_{\left\{T_{1} \circ \tau(s)>u\right\}} \cdot \mathbf{1}_{\left\{\boldsymbol{\ell}_{u}=\mathbf{0}\right\}}[\varphi(1)-\varphi(0)] \alpha N \mid \mathcal{F}_{u}^{Y}\right] \\
& =\mathbf{1}_{\left\{Y_{u}=0\right\}} \cdot[\varphi(1)-\varphi(0)] \alpha N \cdot \mathbb{P}\left(T_{1} \circ \tau(s)>u, \boldsymbol{\ell}_{u}=\mathbf{0} \mid \mathcal{F}_{u}^{Y}\right) \\
& =\mathbf{1}_{\left\{Y_{u}=0\right\}} \cdot[\varphi(1)-\varphi(0)] \alpha N \cdot \mathbb{P}\left(\boldsymbol{\ell}_{u}=\mathbf{0} \mid\left\{T_{1} \circ \tau(s)>u\right\} \cap \mathcal{F}_{u}^{Y}\right) \cdot \mathbb{P}\left(T_{1} \circ \tau(s)>u \mid \mathcal{F}_{u}^{Y}\right) \\
& =\mathbf{1}_{\left\{Y_{u}=0\right\}} \cdot[\varphi(1)-\varphi(0)] \alpha N c^{N} \cdot \mathbb{P}\left(T_{1} \circ \tau(s)>u \mid \mathcal{F}_{u}^{Y}\right) .
\end{aligned}
$$

The first equality is obtained by using the observation that $\mathbf{1}_{\left\{\eta^{-}(\mathbf{x})=0\right\}} \cdot A^{\ell} h \circ g(\mathbf{x})=0$ for all $\mathbf{x}$ but $\mathbf{x}=\mathbf{0}$, where it equals $\varphi(1)-\varphi(0)$. The last equality uses the property that conditionally on $\eta^{-}(\ell)=0$, the process $\eta^{+}(\ell)$ is binomial with parameter $1-c$ and $\left\{\boldsymbol{\ell}_{u}=\mathbf{0}\right\}=\left\{\eta^{+}(\boldsymbol{\ell})=0\right\}$.

Secondly, we suppose $Y_{u}>0$. This implies that $n^{-}\left(\boldsymbol{\ell}_{u}\right)=Y_{u}, n^{0}\left(\boldsymbol{\ell}_{u}\right)=N-Y_{u}$, and $n^{+}\left(\boldsymbol{\ell}_{u}\right)=0$. We get

$$
\begin{aligned}
& \mathbf{1}_{\left\{Y_{u}>0\right\}} \cdot \mathbb{E}\left[\mathbf{1}_{\left\{T_{1} \circ \tau(s)>u\right\}} A^{\ell} f\left(\boldsymbol{\ell}_{u}\right) \mid \mathcal{F}_{u}^{Y}\right] \\
& =\mathbf{1}_{\left\{Y_{u}>0\right\}} \cdot \mathbb{P}\left(T_{1} \circ \tau(s)>u \mid \mathcal{F}_{u}^{Y}\right)\left(N-Y_{u}\right)\left\{\left[\varphi\left(Y_{u}-1\right)-\varphi\left(Y_{u}\right)\right] \gamma+\left[\varphi\left(Y_{u}+1\right)-\varphi\left(Y_{u}\right)\right] \alpha\right\} .
\end{aligned}
$$

Putting together the two expressions, we have:

$$
\mathbb{E}\left[\varphi\left(Y_{t \wedge\left(T_{1} \circ \tau(s)\right)}\right)-\varphi\left(Y_{s}\right) \mid \mathcal{F}_{s}^{Y}\right]=\mathbb{E}\left[\int_{s}^{t} \mathbb{P}\left(T^{1} \circ \tau(s)>u \mid \mathcal{F}_{u}^{Y}\right) \cdot A^{\ell} \varphi\left(Y_{u}\right) d u \mid \mathcal{F}_{s}^{Y}\right] .
$$

Using the strong Markov property and the above result recursively, we can show that for any $n>0$ we have:

$$
\mathbb{E}\left[\varphi\left(Y_{t \wedge T_{n}}\right)-\varphi\left(Y_{s}\right) \mid \mathcal{F}_{s}^{Y}\right]=\mathbb{E}\left[\int_{s}^{t} \mathbb{P}\left(T_{n}>u \mid \mathcal{F}_{u}^{Y}\right) \cdot A^{\ell} \varphi\left(Y_{u}\right) d u \mid \mathcal{F}_{s}^{Y}\right]
$$

Taking the limit as $n$ goes to infinity, using the dominated convergence theorem and the fact that there are finitely many interactions (i.e., times of exit from $I$ ) in any given interval $[s, t]$ we deduce that $\mathbb{P}\left(T^{n}>u \mid \mathcal{F}_{u}^{Y}\right) \rightarrow 1$ as $n \rightarrow \infty$. We conclude that indeed, equation 14 is verified for any $s, t$ with $s \leq t$.

A.3. Proof of Theorem 3.5. As conditionally on the filtration $\left(\mathcal{F}_{t}^{Y}\right)$ all entities in the coalition have the same distribution, it follows that $\mathbb{P}\left(L_{t}^{(N)}(i) \in\{0,1\} \mid \mathcal{F}_{t}^{Y}\right)=\frac{N-Y_{t}^{(N)}}{N}$. Consequently:

$$
\begin{aligned}
h(n, N) & =\mathbb{E}\left[\int_{0}^{\infty} \mathbb{P}\left(L_{t}^{(N)}(i) \in\{0,1\} \mid \mathcal{F}_{t}^{Y}\right) d t \mid Y_{0}^{(N)}=n\right] \\
& =\mathbb{E}\left[\int_{0}^{\infty} \frac{N-Y_{t}^{(N)}}{N} d t \mid Y_{0}^{(N)}=n\right] .
\end{aligned}
$$


The functions $h(n, N), n \in\{0, \ldots, N\}$ satisfy the following system of equations:

$$
\left\{\begin{array}{l}
\sum_{k=0}^{N}(h(k, N)-h(n, N)) q^{(N)}(n, k)=-\frac{N-n}{N} \\
h(N, N)=0
\end{array}\right.
$$

that is:

$$
\left\{\begin{array}{l}
(h(1, N)-h(0, N)) \alpha N c^{N}=-1, \\
(h(n+1, N)-h(n, N)) \alpha(N-n)+(h(n-1, N)-h(n, N)) \gamma(N-n)=-\frac{N-n}{N}, \\
h(N, N)=0 .
\end{array}\right.
$$

Rearranging terms, we obtain:

$$
\left\{\begin{array}{l}
\alpha h(n+1, N)-(\alpha+\gamma) h(n, N)+\gamma h(n-1, N)+\frac{1}{N}=0 \\
h(1, N)=h(0, N)-\frac{1}{N \alpha c^{N}} \\
h(N, N)=0
\end{array}\right.
$$

This is a second-order linear recursive equation. By defining $\Delta(n, N)=N(h(n, N)-h(n+1, N))$, we can transform the equations (20) into a first-order linear difference equation as:

$$
\left\{\begin{array}{l}
\Delta(n, N)=\frac{1}{\alpha}+\frac{\gamma}{\alpha} \Delta(n-1, N), \\
\Delta(0, N)=\frac{1}{\alpha c^{N}}
\end{array}\right.
$$

with solution: for $n=0,1, \cdots, N$

$$
\Delta(n, N)= \begin{cases}\frac{1}{\alpha c^{N}}+\frac{n}{\alpha} & , \text { if } \gamma=\alpha, \\ \left(\frac{1}{\alpha c^{N}}-\frac{1}{\alpha-\gamma}\right)\left(\frac{\gamma}{\alpha}\right)^{n}+\frac{1}{\alpha-\gamma} & , \text { otherwise. }\end{cases}
$$

By the telescoping property of the difference sequence (and the fact that $h(N, N)=0$ ), we have that

$$
h(n, N)=h(n, N)-h(N, N)=\frac{1}{N} \sum_{k=n}^{N-1} \Delta(k, N),
$$

and the result follows from equation (21).

Remark A.6. An alternative representation of $h(n, N)$ obtained also from 22)-(21), which has the benefit of working for both $\alpha=\gamma$ and $\alpha \neq \gamma$, is:

$$
h(n, N)=\frac{1}{\alpha N c^{N}} \sum_{k=n}^{N-1}\left(\xi^{k}+c^{N} \sum_{j=0}^{k-1} \xi^{j}\right) .
$$

We conclude by a property that follows directly form (23), and that will be useful later on:

Lemma A.7. The mapping $n \mapsto h(n, N)$ is strictly decreasing. 
A.4. Proof of Proposition 3.6. Let $X \sim \operatorname{Bin}(p, N)$ so that $\mathbb{E}[X]=N p$. We observe that when $\xi=\frac{\gamma}{\alpha}=1$, $\mathbb{E}[h(X, N)]$ diverges to $+\infty$ as $N \rightarrow+\infty$. We now study the case $\xi \neq 1$. Using Newton's binomial, we obtain:

$$
\mathbb{E}\left[\xi^{X}\right]=\sum_{n=0}^{N} \xi^{n}\left(\begin{array}{l}
N \\
n
\end{array}\right) p^{n}(1-p)^{N-n}=(1-p+p \xi)^{N}
$$

Using the representation of the function $h$ in Theorem 3.5 , we obtain:

$$
\begin{aligned}
\mathbb{E}[h(X, N)] & =\frac{1-\mathbb{E}[X] / N}{\alpha-\gamma}+\frac{1}{\alpha N c^{N}}\left(1-\frac{c^{N}}{1-\xi}\right)\left(\frac{\mathbb{E}\left[\xi^{X}\right]-\xi^{N}}{1-\xi}\right) \\
& =h^{*}(p)+\left[\frac{1}{\alpha N c^{N}}\left(1-\frac{c^{N}}{1-\xi}\right)\left(\frac{(1-p+p \xi)^{N}-\xi^{N}}{1-\xi}\right)\right]
\end{aligned}
$$

If $\xi>1$ the expression diverges to $+\infty$ as $N \rightarrow \infty$. If $\xi<1$ this expression is not necessarily convergent since $c^{N} \rightarrow 0$ as $N \rightarrow \infty$ and it appears at the denominator. Nevertheless, ignoring terms that vanish asymptotically, we have that the term in the square brackets behaves as

$$
\frac{1}{\alpha N c^{N}}\left(1-\frac{c^{N}}{1-\xi}\right)\left(\frac{(1-p+p \xi)^{N}-\xi^{N}}{1-\xi}\right) \sim \frac{1}{\alpha N(1-\xi)}\left(\frac{1-p+p \xi}{c}\right)^{N}
$$

which converges (to zero) if and only if $c \geq 1-p+p \xi$. As $c, \xi \in(0,1)$, this last condition is equivalent to $\xi \leq c$ and $p \in\left[\frac{1-c}{1-\xi}, 1\right]$.

A.5. Proof of Theorem 3.7. As the entities interact, they are interdependent and the limiting process cannot be computed using the strong law of large numbers. We employ the theory presented in Ethier and Kurtz [12]). We first write the process $Z_{t}^{(N)}:=\frac{Y_{t}^{(N)}}{N}$ in the form of a stochastic differential equation (cf. Section 10.4 in [12]. So, for now, $N$ is fixed. We define two counting processes $P_{t}^{+(N)}$ and $P_{t}^{-(N)}$. The process $P_{t}^{+(N)}$ counts the positive jumps of the process $Y_{t}^{(N)}$ and the process $P_{t}^{-(N)}$ counts the negative jumps of the process $Y_{t}^{(N)}$, so that:

$$
Y_{t}^{(N)}=Y_{0}^{(N)}+P_{t}^{+(N)}-P_{t}^{-(N)} .
$$

It follows from the transition matrix of the process $Y^{(N)}$ that is given in Theorem 3.2, that the predictable compensator of the process $P_{t}^{+(N)}$ is given by process

$$
\int_{0}^{t} q^{(N)}\left(\left(Y_{s}^{(N)}\right), i+1\right) \mathbf{1}_{\left\{Y_{s}^{(N)}<N\right\}} d s=\int_{0}^{t} \alpha\left(N-Y_{s}^{(N)}\right) d s
$$

and the predictable compensator of the process $P_{t}^{-(N)}$ is the process is

$$
\int_{0}^{t} q^{(N)}\left(\left(Y_{s}^{(N)}\right), i-1\right) \mathbf{1}_{\left\{Y_{s}^{(N)}>0\right\}} d s=\int_{0}^{t} \gamma\left(N-Y_{s}^{(N)}\right) \mathbf{1}_{\left\{Y_{s}^{(N)}>0\right\}} d s
$$


Therefore, the following processes are martingales:

$$
M_{t}^{+(N)}=P_{t}^{+(N)}-\int_{0}^{t} \beta^{+(N)}\left(Y_{s}^{(N)}\right) d s, \text { and } M_{t}^{-(N)}=P_{t}^{-(N)}-\int_{0}^{t} \beta^{-(N)}\left(Y_{s}^{(N)}\right) d s,
$$

where $\beta^{+(N)}(i)=q^{(N)}(i, i+1), \quad \beta^{-(N)}(i)=q^{(N)}(i, i-1) \mathbf{1}_{\{i>0\}}$. We denote $M_{t}^{(N)}:=M_{t}^{+(N)}-M_{t}^{-(N)}$, which is a martingale and $\beta^{(N)}:=\beta^{+(N)}-\beta^{-(N)}$ and we obtain $Y_{t}^{(N)}=Y_{0}^{(N)}+M_{t}^{(N)}-\int_{0}^{t} \beta^{(N)}\left(Y_{s}^{(N)}\right) d s$. Therefore, $Z^{(N)}$ satisfies the following SDE:

$$
Z_{t}^{(N)}=Z_{0}^{(N)}+\frac{M_{t}^{(N)}}{N}-\int_{0}^{t} \frac{\beta^{(N)}\left(N Z_{s}^{(N)}\right)}{N} d s .
$$

To calculate the limit of the process $Z^{(N)}$ for $N \rightarrow \infty$, we compute the limit of each term in the left hand side of 25 . The martingale $\frac{M_{t}^{(N)}}{N}$ is a finite variation martingale bounded, because it takes values in $\left.[0,1]\right)$, and with jumps of size $\pm \frac{1}{N}$. We have that the relation (10.1) in the Theorem 10.5 from [19] is satisfied:

$$
\frac{1}{N} \sup _{s \leq t}\left|M_{s}^{(N)}-M_{s-}^{(N)}\right| \leq \frac{1}{N} \underset{N \rightarrow \infty}{\longrightarrow} 0 .
$$

The quadratic variation of $\frac{M_{t}^{(N)}}{N}$ is the sum of the square of its jumps:

$$
\left[M^{(N)}\right]_{t} /\left(N^{2}\right)=\left(P_{t}^{+(N)}+P_{t}^{-(N)}\right) /\left(N^{2}\right)
$$

and:

$$
\mathbb{E}\left[\left[M^{(N)}\right]_{t}\right] / N^{2} \underset{N \rightarrow \infty}{\longrightarrow} 0 .
$$

Indeed, $\beta^{+(N)}\left(Y_{t}^{(N)}\right) \leq \alpha N$ and $\beta^{-(N)}\left(Y_{t}^{(N)}\right) \leq \gamma N$, therefore using 24, we obtain:

$$
\begin{aligned}
& \mathbb{E}\left[P_{t}^{+(N)}\right]=\mathbb{E}\left[\int_{0}^{t} \beta^{+(N)}\left(Y_{s}^{(N)}\right) d s\right] \leq \alpha t N, \\
& \mathbb{E}\left[P_{t}^{-(N)}\right]=\mathbb{E}\left[\int_{0}^{t} \beta^{-(N)}\left(Y_{s}^{(N)}\right) d s\right] \leq \gamma t N
\end{aligned}
$$

Hence, from Theorem 10.5 and Remark 10.6 from [19] (see also Theorem 7.1.4 in [12]) we have that $\frac{M_{t}^{(N)}}{N} \rightarrow 0$, when $N$ tends to infinity. It remains to compute the limit when $N$ tends to infinity for $\int_{0}^{t} \frac{\beta^{(N)}\left(N Z_{s}^{(N)}\right)}{N} d s$, the third term in the expression 25 of $Z^{(N)}$. We write the transition intensities as 
functions of $z:=\frac{i}{N}$ and $N$ using the Theorem 3.2, we have:

$$
\begin{aligned}
& \frac{\beta^{+(N)}(N z)}{N}=\frac{q^{(N)}(i, i+1)(i)}{N}=\alpha \frac{c^{N}}{N} \underset{N \rightarrow \infty}{\longrightarrow} 0, \quad \text { if } z=0, \\
& \frac{\beta^{+(N)}(N z)}{N}=\frac{q^{(N)}(i, i+1)(i)}{N}=\alpha(1-z), \quad \text { if } z \in\left\{\frac{1}{N}, \cdots, \frac{N-1}{N}, 1\right\}, \\
& \frac{\beta^{-(N)}(N z)}{N}=\frac{q^{(N)}(i, i-1)(i)}{N}=\gamma(1-z), \quad \text { if } z \in\left\{\frac{1}{N}, \cdots, \frac{N-1}{N}, 1\right\}, \\
& \frac{\beta^{-(N)}(N z)}{N}=0, \quad \text { if } z=0 .
\end{aligned}
$$

It follows that:

$$
\int_{0}^{t} \frac{\beta^{(N)}\left(N Z_{s}^{(N)}\right)}{N} d s=\int_{0}^{t} \frac{\left(\beta^{+(N)}+\beta^{-(N)}\right)\left(N Z_{s}^{(N)}\right)}{N} d s \underset{N \rightarrow \infty}{\rightarrow} \int_{0}^{t \wedge \tau}\left(1-Z_{s}\right)(\alpha-\gamma) d s,
$$

where $\tau=\inf \left\{t \mid Z_{t}=0\right\}$. Finally, supposing $z_{0}=\lim _{N \rightarrow \infty} Y_{0}^{(N)} / N$ exists, we obtain $Z_{t}:=\lim _{N \rightarrow \infty} Z_{t}^{(N)}$ is given by:

and the result follows.

$$
Z_{t}=z_{0}-\int_{0}^{t \wedge \tau}\left(1-Z_{s}\right)(\alpha-\gamma) d s
$$

\section{REFERENCES}

[1] ÁBRAHÁM, A AND A. LACZÓ (2018): “Efficient Risk Sharing with Limited Commitment and Storage”, Review of Economic Studies, 85(3), 1389-1424.

[2] AKERLOF, G. (1970): "The Market for "Lemons": Quality Uncertainty and the Market Mechanism," Quarterly Journal of Economics, 84, 488-500.

[3] ATTANASIO, O., A. BARR, J.C. CARDENAS, G. GENICOT and C. MEGHIR (2012): "Risk Pooling, Risk Preferences, and Social Networks", American Economic Journal: Applied Economics, 4 (2), 134-167.

[4] BALA, V., and S. GOYAL (2000): "A Non-cooperative Model of Network Formation," Econometrica, 68 (5), 1181-229.

[5] BARR, A., AND G. GENICOT (2008): "Risk Sharing, Commitment, and Information: An Experimental Analysis." Journal of the European Economic Association, 6 (6). 1151-85.

[6] BESLEY, T. AND S. COATE (1995): “Group Lending, Repayment Incentives, and Social Collateral,” J. Devel. Econ., 46 (1), 1-18.

[7] BRAMOULLÉ, Y. AND R. KRANTON (2007): "Risk-Sharing Networks," Journal of Economic Behavior and Organization, 64 (3-4): 275-294.

[8] COATE, S., AND M. RAVALLION (1993): "Reciprocity without Commitment: Characterisations and Performance of Informal Risk-sharing Arrangements," Journal of Development Economics, 40, 1-24.

[9] COLE, H.L. AND N.R. KOCHERLAKOTA (2001): "Efficient Allocations with Hidden Income and Hidden Storage", Review of Economic Studies, 68, 523-542.

[10] COX, D. (1987): "Motives for Private Income Transfers," J. Pol. Econ., 95 (3), 508-543.

[11] DEATON, A. (1992): "Understanding Consumption," Oxford: Clarendon Press.

[12] ETHIER N.S., and T.G. KURTZ (1986): "Markov Processes-Characterization and Convergence", Willey-Interscience.

[13] FAFCHAMPS, M., AND S. LUND (2002): "Risk-Sharing Networks in Rural Philippines," Journal of Development Economics, 71 (2), 261-287. 
[14] GENICOT, G., AND D. RAY (2003): “Group Formation in Risk-Sharing Arrangements," Review of Economic Studies, 70 (1), 87-113.

[15] GRIMARD F. (1997): "Household Consumption Smoothing through Ethnic Ties: Evidence from Côte d'Ivoire," Journal of Development Economics, 53, 391-421.

[16] KARATZAS, I., AND S. SHREVE (1998): "Brownian Motion and Stochastic Calculus", Springer.

[17] KIMBALL, M. (1988): "Farmers' cooperatives as behavior toward risk," American Economic Review, 78 (1) $224-232$.

[18] KOCHERLAKOTA, N. (1996): "Implications of Efficient Risk Sharing without Commitment," Review of Economic Studies, 63, 595-609.

[19] KURTZ T.G. (2001): "Lectures on Stochastic Analysis," University of Wisconsin-Madison.

[20] LIGON E., J. THOMAS, AND T. WORRALL (2002): "Mutual Insurance and Limited Commitment: Theory and Evidence in Village Economies", Review of Economic Studies, 69, 115-139.

[21] MÉLÉARD, S., AND D. VILLEMONAIS (2012): "Quasi-stationary distributions and population processes," Probab. Surveys, 9, 340-410.

[22] MORDUCH, J. (2002): "Consumption Smoothing Across Space: Tests for Village Level Response to Risk," Discussion Paper No. 2002/55, Harvard University.

[23] MURGAI, R., P. WINTERS, E. SADOULET, AND A. DE JANVRY (2002): "Localized and incomplete mutual insurance", Journal of Development Economics, 67 (2), 245-274.

[24] NEYMAN, A. (2017): "Continuous-Time Stochastic Games," Games and Economic Behavior, 104, 92-130.

[25] RAVALLION, M., and S. Chaudhuri (1997). "Risk and Insurance in Village India: Comment." Econometrica, 65, $171-184$.

[26] RAVALLION, M., and L. DEARDEN (1988). "Social Security in a "Moral Economy": An Empirical Analysis for Java." Review of Economics and Statistics, 70, 36-44.

[27] REVUZ D., AND M. YOR (1999): “Continuous Martingales and Brownian Motion,” Springer, Third edition.

[28] SADOULET, L. (2000): "Equilibrium Risk-Matching in Group Lending.” Econometric Society World Congress 2000, Contributed Papers 1302.

[29] TOWNSEND, R. (1994), "Risk and Insurance in Village India," Econometrica, 62, 539-591.

[30] UDRY C. (1994), "Risk and Insurance in a Rural Credit Market: An Empirical Investigation in Northern Nigeria" Review of Economic Studies, 6, 495-526. 